\title{
Boundedness Criteria and Norm of Some Multilinear Hilbert-Type Operators
}

\author{
Justice S. Bansah and Benoît F. Sehba \\ Department of Mathematics, University of Ghana, P.O. Box LG 62, Legon, Accra, Ghana \\ Correspondence should be addressed to Benoît F. Sehba; bsehba@gmail.com
}

Received 15 October 2016; Accepted 11 January 2017; Published 8 February 2017

Academic Editor: Wing-Sum Cheung

Copyright (C) 2017 Justice S. Bansah and Benoît F. Sehba. This is an open access article distributed under the Creative Commons Attribution License, which permits unrestricted use, distribution, and reproduction in any medium, provided the original work is properly cited.

We consider two families of multilinear Hilbert-type operators for which we give exact relations between the parameters so that they are bounded. We also find the exact norm of these operators.

\section{Introduction}

Let $1 \leq p<\infty$, and let $t$ be a real number. We denote by $L_{t}^{p}((0, \infty))$ or simply $L_{t}^{p}$ the weighted Lebesgue space $L^{p}\left((0, \infty), y^{t} d y\right)$. When $t=0$, we simply write $L^{p}$ for the corresponding space. We will be using the notation

$$
\|f\|_{p, t}:=\left(\int_{0}^{\infty}|f(y)|^{p} y^{t} \mathrm{~d} y\right)^{1 / p}
$$

and we recall that $f \in L_{t}^{p}((0, \infty))$ if and only if the above quantity is finite. For $t=0$, we simply write $\|f\|_{p}$ for $\|f\|_{p, 0}$. All over the text, for $1 \leq p \leq \infty$ we denote by $p^{\prime}$ its conjugate exponent, that is, the unique extended real number satisfying $1=1 / p+1 / p^{\prime}$.

We recall that the Hilbert operator is defined by

$$
H f(x):=\int_{0}^{\infty} \frac{f(y)}{x+y} \mathrm{~d} y .
$$

The boundedness of this operator has been heavily studied in the literature; in particular people have been very interested in the norm estimate of this operator and its siblings (see, e.g., the following and the references therein [1-6]). In [7], we considered a more general family of this operator for which we provided boundedness criteria and some sharp norm estimates. More precisely, for $\beta, \gamma$ real parameters we considered the family of operators $H_{\beta, \gamma}$ defined for compactly supported functions by

$$
H_{\beta, \gamma} f(x):=\int_{0}^{\infty} \frac{f(y)}{(x+y)^{\gamma}} y^{\beta} \mathrm{d} y .
$$

This family as shown in [7] can be related to Bergman-type projections. This family can be extended in two different ways into $m$-linear operators on $(0, \infty)^{m}$. For the first family, we let $\beta_{1}, \ldots, \beta_{m}, \gamma_{1}, \ldots, \gamma_{m}$ be real parameters and put $\vec{\gamma}=$ $\left(\gamma_{1}, \ldots, \gamma_{m}\right)$ and $\vec{\beta}=\left(\beta_{1}, \ldots, \beta_{m}\right)$. Put $\vec{f}=\left(f_{1}, \ldots, f_{m}\right)$, where $f_{j}, j=1, \ldots, m$, are compactly supported functions on $(0, \infty)$. Consider the operators $T_{\vec{\beta}, \vec{\gamma}}$ defined by

$$
\begin{aligned}
& T_{\vec{\beta}, \vec{\gamma}}(\vec{f})(x) \\
& \quad:=\int_{(0, \infty)^{m}} \frac{f_{1}\left(y_{1}\right) f_{2}\left(y_{2}\right) \cdots f_{m}\left(y_{m}\right)}{\left(x+y_{1}\right)^{\gamma_{1}}\left(x+y_{2}\right)^{\gamma_{2}} \cdots\left(x+y_{m}\right)^{\gamma_{m}}} y_{1}^{\beta_{1}} y_{2}^{\beta_{2}} \\
& \quad \cdots y_{m}^{\beta_{m}} \mathrm{~d} y_{1} \mathrm{~d} y_{2} \cdots \mathrm{d} y_{m} .
\end{aligned}
$$

Following the idea in [7], one can also relate this family to multilinear Bergman projections. To define the second 
family, we let $\beta_{1}, \ldots, \beta_{m}, \gamma$ be real parameters and put $\vec{\beta}=$ $\left(\beta_{1}, \ldots, \beta_{m}\right)$. We define the operators $S_{\vec{\beta}, \gamma}$ as follows:

$$
\begin{aligned}
& S_{\vec{\beta}, \gamma}(\vec{f})(x) \\
& \quad=\int_{(0, \infty)^{m}} \frac{f_{1}\left(y_{1}\right) f_{2}\left(y_{2}\right) \cdots f_{m}\left(y_{m}\right)}{\left(x+y_{1}+\cdots+y_{m}\right)^{\gamma}} y_{1}^{\beta_{1}} y_{2}^{\beta_{2}} \\
& \quad \cdots y_{m}^{\beta_{m}} \mathrm{~d} y_{1} \mathrm{~d} y_{2} \cdots \mathrm{d} y_{m} .
\end{aligned}
$$

This last family appears in [8] in the study of the Laplace representation of some mixed norm Bergman spaces in relation to the question of the boundedness of the Bergman projection in tube domains over symmetric cones. Many authors have provided the norm of the operators $S_{\vec{\beta}, \gamma}$ in some particular cases and some variations [9-14]. The fact is that, in these papers, the relations between the parameters are directly given without indicating how they are obtained and the authors are only interested in finding the exact norm of the operators or proving the corresponding Hardy-Hilbert inequality.

We aim in this note to provide exact relations between the parameters so that the above operators are bounded from $L_{t_{1}}^{p_{1}} \times \cdots \times L_{t_{m}}^{p_{m}}$ to $L_{t}^{p}$ when $1 \leq p_{1}, \ldots, p_{m} \leq \infty, t_{1}, \ldots, t_{m}, t$ being real numbers with

$$
\begin{aligned}
& \frac{1}{p_{1}}+\cdots+\frac{1}{p_{m}}=\frac{1}{p}, \\
& \frac{t_{1}}{p_{1}}+\cdots+\frac{t_{m}}{p_{m}}=\frac{t}{p} .
\end{aligned}
$$

We also find the exact norm of these operators, extending the results in $[9,12]$. Note that, in the above relations, we allow some (not all) of the exponents $p_{j}$ s to be infinite, a situation which has not been considered before as far as we know.

\section{Statement of the Results}

We give in this section all our results. We denote, by $B(x, y)$, the Beta-function of $x$ and $y$ to be defined in the next section. We start by the following.

Theorem 1. Let $\beta_{1}, \ldots, \beta_{m}, \gamma_{1}, \ldots, \gamma_{m}$ be real numbers. Let $1<$ $p_{1}, \ldots, p_{m}<\infty, 1 \leq p<\infty$, let $t_{1}, \ldots, t_{m}$, $t$ be real numbers, and assume that (6) and (7) hold. Then the following conditions are equivalent.

(i) The operator $T_{\vec{\beta}, \vec{\gamma}}$ is bounded from $L_{t_{1}}^{p_{1}}((0, \infty)) \times \cdots \times$ $L_{t_{m}}^{p_{m}}((0, \infty))$ to $L_{t}^{p}((0, \infty))$.

(ii) The parameters satisfy

$$
\sum_{j=1}^{m} \gamma_{j}=\sum_{j=1}^{m} \beta_{j}+m
$$

$$
-p_{j}\left(\gamma_{j}-\beta_{j}-1\right)<t_{j}+1<p_{j}\left(\beta_{j}+1\right)
$$

$$
j=1, \ldots, m \text {. }
$$

In this case, if we denote by $\left\|T_{\vec{\beta}, \vec{\gamma}}\right\|_{\left(\prod_{j=1}^{m} L_{t_{j}}^{p_{j}}\right) \rightarrow L_{t}^{p_{m}}}$ the operator norm of $T_{\vec{\beta}, \vec{\gamma}}$, then

$$
\begin{aligned}
& \left\|T_{\vec{\beta}, \vec{\gamma}}\right\|_{\left(\prod_{j=1}^{m} L_{t_{j}}^{p_{j}}\right) \rightarrow L_{t}^{p}} \\
& \quad=\prod_{j=1}^{m} B\left(\beta_{j}+1-\frac{t_{j}+1}{p_{j}}, \gamma_{j}-\beta_{j}-1+\frac{t_{j}+1}{p_{j}}\right) .
\end{aligned}
$$

It is easy to see that condition (9) provides that, for any $j \in\{1, \ldots, m\}$,

$$
\gamma_{j}>0
$$

hence

$$
\sum_{j=1}^{m} \gamma_{j}>0
$$

As $-p_{j}\left(\gamma_{j}-\beta_{j}-1\right)<t_{j}+1, j=1, \ldots, m$, we also have that

$$
\sum_{j=1}^{m} \gamma_{j}>\sum_{j=1}^{m}\left(\beta_{j}+1-\frac{t_{j}+1}{p_{j}}\right)
$$

Now observing that (8) can be written as

$$
\sum_{j=1}^{m} \gamma_{j}=\sum_{j=1}^{m}\left(\beta_{j}+1-\frac{t_{j}+1}{p_{j}}\right)+\frac{t+1}{p}
$$

we conclude that $t+1>0$ or equivalently that $t>-1$.

We also obtain the following result.

Theorem 2. Let $\beta_{1}, \ldots, \beta_{m}, \gamma$ be real numbers. Let $1<$ $p_{1}, \ldots, p_{m}<\infty, 1 \leq p<\infty$, let $t_{1}, \ldots, t_{m}$, $t$ be real numbers, and assume that (6) and (7) hold. Then the following conditions are equivalent.

(i) The operator $S_{\vec{\beta}, \gamma}$ is bounded from $L_{t_{1}}^{p_{1}}((0, \infty)) \times \cdots \times$ $L_{t_{m}}^{p_{m}}((0, \infty))$ to $L_{t}^{p}((0, \infty))$.

(ii) The parameters satisfy

$$
\begin{gathered}
0<\gamma=\sum_{j=1}^{m} \beta_{j}+m, \quad t>-1, \\
t_{j}+1<p_{j}\left(\beta_{j}+1\right), \quad j=1, \ldots, m .
\end{gathered}
$$


In this case, if we denote by $\left\|S_{\vec{\beta}, \gamma}\right\|_{\left(\prod_{j=1}^{m} L_{t_{j}}^{p_{j}}\right) \rightarrow L_{t}^{p_{m}}}$ the operator norm of $S_{\vec{\beta}, \gamma}$, then

$$
\begin{aligned}
& \left\|S_{\vec{\beta}, \gamma}\right\|_{\left(\prod_{j=1}^{m} L_{t_{j}}^{p_{j}}\right) \rightarrow L_{t}^{p}} \\
& =\frac{\left(\prod_{j=1}^{m} \Gamma\left(\beta_{j}+1-\left(t_{j}+1\right) / p_{j}\right)\right) \Gamma((t+1) / p)}{\Gamma(\gamma)} .
\end{aligned}
$$

We note that the norm of the operator $S_{\vec{\beta}, \gamma}$ for $\vec{\beta}=\overrightarrow{0}$ and $\gamma=m$ was computed in [9] for the unweighted case and [12] for the weighted case.

If in relation (6) we allow only some (but not all) of the exponents to be finite while all the other exponents are equal to infinity, then we obtain a kind of mixed endpoints version of the previous results.

Theorem 3. Let $\beta_{1}, \ldots, \beta_{m}, \gamma_{1}, \ldots, \gamma_{m}$ be real numbers. Let $1<p_{1}, \ldots, p_{l}<\infty, 1 \leq l<m, 1 \leq p<\infty$, and assume that $1 / p_{1}+\cdots+1 / p_{l}=1 / p$. Let $t_{1}, \ldots, t_{l}, t$ be real numbers and assume that $t_{1} / p_{1}+\cdots+t_{l} / p_{l}=t / p$. Then the following conditions are equivalent.

(i) The operator $T_{\vec{\beta}, \vec{\gamma}}$ is bounded from $L_{t_{1}}^{p_{1}}((0, \infty)) \times \cdots \times$ $L_{t_{l}}^{p_{l}}((0, \infty)) \times\left(L^{\infty}((0, \infty))\right)^{m-l}$ to $L_{t}^{p}((0, \infty))$.

(ii) The parameters satisfy

$$
\begin{gathered}
\sum_{j=1}^{m} \gamma_{j}=\sum_{j=1}^{m} \beta_{j}+m ; \\
-p_{j}\left(\gamma_{j}-\beta_{j}-1\right)<t_{j}+1<p_{j}\left(\beta_{j}+1\right),
\end{gathered}
$$

$$
\begin{array}{r}
\text { for } j=1, \ldots, l, \\
\gamma_{j}>\beta_{j}+1>0, \quad \text { for } j=l+1, \ldots, m .
\end{array}
$$

In this case, if we denote by $\left\|T_{\vec{\beta}, \vec{\gamma}}\right\|_{\left(\prod_{j=1}^{l} L_{t_{j}}^{p_{j}}\right) \times\left(L^{\infty}\right)^{m-l} \rightarrow L_{t}^{p}}$ the operator norm of $T_{\vec{\beta}, \vec{\gamma}}$, then

$$
\begin{aligned}
&\left\|T_{\vec{\beta}, \vec{\gamma}}\right\|_{\left(\prod_{j=1}^{l} L_{t_{j}}^{p_{j}}\right) \times\left(L^{\infty}\right)^{m-l} \rightarrow L_{t}^{p}} {\left[\prod_{j=1}^{l} B\left(\beta_{j}+1-\frac{t_{j}+1}{p_{j}}, \gamma_{j}-\beta_{j}-1+\frac{t_{j}+1}{p_{j}}\right)\right] } \\
& \quad \times\left[\prod_{j=l+1}^{m} B\left(\beta_{j}+1, \gamma_{j}-\beta_{j}-1\right)\right] .
\end{aligned}
$$

We also obtain the following result.

Theorem 4. Let $\beta_{1}, \ldots, \beta_{m}, \gamma_{1}, \ldots, \gamma_{m}$ be real numbers. Let $1<p_{1}, \ldots, p_{l}<\infty, 1 \leq l<m, 1 \leq p<\infty$, and assume that $1 / p_{1}+\cdots+1 / p_{l}=1 / p$. Let $t_{1}, \ldots, t_{l}, t$ be real numbers and assume that $t_{1} / p_{1}+\cdots+t_{l} / p_{l}=t / p$. Then the following conditions are equivalent.

(i) The operator $S_{\vec{\beta}, \gamma}$ is bounded from $L_{t_{1}}^{p_{1}}((0, \infty)) \times \cdots \times$ $L_{t_{l}}^{p_{l}}((0, \infty)) \times\left(L^{\infty}((0, \infty))\right)^{m-l}$ to $L_{t}^{p}((0, \infty))$.

(ii) The parameters satisfy

$$
\begin{gathered}
0<\gamma=\sum_{j=1}^{m} \beta_{j}+m, \quad t>-1 \\
t_{j}+1<p_{j}\left(\beta_{j}+1\right), \quad \text { for } j=1, \ldots, l, \\
\beta_{j}>-1, \quad \text { for } j=l+1, \ldots, m .
\end{gathered}
$$

In this case, if we denote by $\left\|S_{\vec{\beta}, \gamma}\right\|_{\left(\prod_{j=1}^{l} L_{t_{j}}^{p_{j}}\right) \times\left(L^{\infty}\right)^{m-l} \rightarrow L_{t}^{p}}$ the operator norm of $S_{\vec{\beta}, \gamma}$, then

$$
\left\|S_{\vec{\beta}, \gamma}\right\|_{\left(\prod_{j=1}^{l} L_{t_{j}}^{p_{j}}\right) \times\left(L^{\infty}\right)^{m-l} \rightarrow L_{t}^{p}}=\frac{\left(\prod_{j=1}^{l} \Gamma\left(\beta_{j}+1-\left(t_{j}+1\right) / p_{j}\right)\right) \Gamma((t+1) / p)}{\Gamma(\gamma)}\left[\prod_{j=l+1}^{m} \Gamma\left(\beta_{j}+1\right)\right] .
$$

We are essentially motivated by the need of explaining the right relations between the parameters that make these operators bounded and the generalization of the previous results on the norm estimates of these operators. In the proofs of the necessary parts, we appeal to duality and use appropriate local test functions. Note that as our operators are $m$-linear, each of them has $m$ adjoints. For the computation of the norm of each operator, to simplify our presentation, we give an upper estimate and a lower estimate. The proof of the lower estimate appeals to a clever choice of test functions, a good decomposition of multiple integrals to find the right lower bound.

\section{Some Useful Tools}

We recall that the Beta-function of the cone $(0, \infty)$ is defined by

$$
B(m, n)=B(n, m)=\int_{0}^{\infty} \frac{u^{m-1}}{(1+u)^{m+n}} \mathrm{~d} u
$$

We note that this integral converges if and only if $m, n>0$. Recall that

$$
B(m, n)=\frac{\Gamma(m) \Gamma(n)}{\Gamma(n+m)},
$$


where $\Gamma$ is the classical Gamma-function. The following can be obtained by induction (see, e.g., [10, Lemma 5.1]):

$$
\begin{aligned}
& \int_{(0, \infty))^{s-1}} \frac{u_{1}^{m_{1}-1} \cdots u_{s-1}^{m_{s-1}-1}}{\left(1+u_{1}+\cdots+u_{s-1}\right)^{\sum_{j=1}^{s} m_{j}}} \mathrm{~d} u_{1} \cdots \mathrm{d} u_{s} \\
& =\frac{\prod_{j=1}^{s} \Gamma\left(m_{j}\right)}{\Gamma\left(\sum_{j=1}^{s} m_{j}\right)}
\end{aligned}
$$

where $m_{j}>0, j=1, \ldots, s$.

\section{The Norm of $T_{\vec{\beta}, \vec{\gamma}}$ and $S_{\vec{\beta}, \gamma}$}

4.1. The Norm of $T_{\vec{\beta}, \vec{\gamma}}$. We have the following upper estimate of the norm of $T_{\vec{\beta}, \vec{\gamma}}$.

Lemma 5. Let $\beta_{1}, \ldots, \beta_{m}, \gamma_{1}, \ldots, \gamma_{m}$ be real numbers. Let $1<$ $p_{1}, \ldots, p_{m}<\infty, 1 \leq p<\infty$, and let $t_{1}, \ldots, t_{m}$, t be real numbers and assume that relations (6) and (7) hold. Assume that

$$
\begin{gathered}
\sum_{j=1}^{m} \gamma_{j}=\sum_{j=1}^{m} \beta_{j}+m, \\
-p_{j}\left(\gamma_{j}-\beta_{j}-1\right)<t_{j}+1<p_{j}\left(\beta_{j}+1\right), \\
j=1, \ldots, m .
\end{gathered}
$$

Then the operator $T_{\vec{\beta}, \vec{\gamma}}$ is bounded from $L_{t_{1}}^{p_{1}}((0, \infty)) \times \cdots \times$ $L_{t_{m}}^{p_{m}}((0, \infty))$ to $L_{t}^{p}((0, \infty))$. Moreover,

$$
\begin{aligned}
& \left\|T_{\vec{\beta}, \vec{\gamma}}\right\|_{\left(\prod_{j=1}^{m} L_{t_{j}}^{p_{j}}\right) \rightarrow L_{t}^{p}} \\
& \quad \leq \prod_{j=1}^{m} B\left(\beta_{j}+1-\frac{t_{j}+1}{p_{j}}, \gamma_{j}-\beta_{j}-1+\frac{t_{j}+1}{p_{j}}\right) .
\end{aligned}
$$

Proof. For $f_{i} \in L_{t_{i}}^{p_{i}}, i=1, \ldots, m$, we write again $\vec{f}=\left(f_{1}, \ldots, f_{m}\right)$. Using an easy change of variables and Minkowski's inequality, we obtain

$$
\begin{aligned}
& \left\|T_{\vec{\beta}, \vec{p}}(\vec{f})\right\|_{p, t}=\left(\int_{0}^{\infty}\left|T_{\vec{\beta}, \vec{\gamma}}(\vec{f})(x)\right|^{p} x^{t} \mathrm{~d} x\right)^{1 / p} \leq\left[\int_{0}^{\infty}\left(\int_{(0, \infty)^{m}} \frac{\prod_{i=1}^{m}\left|f_{i}\left(y_{i}\right)\right|}{\prod_{i=1}^{m}\left(x+y_{i}\right)^{\gamma_{i}}}\left(\prod_{i=1}^{m} y_{i}^{\beta_{i}}\right) \mathrm{d} y_{1} \mathrm{~d} y_{2} \cdots \mathrm{d} y_{m}\right)^{p} x^{t} \mathrm{~d} x\right]^{1 / p} \\
& =\left[\int_{0}^{\infty}\left(\int_{(0, \infty)^{m}} \frac{\prod_{i=1}^{m}\left|f_{i}\left(u_{i} x\right)\right|}{\prod_{i=1}^{m}\left(1+u_{i}\right)^{\gamma_{i}}}\left(\prod_{i=1}^{m} u_{i}^{\beta_{i}}\right) \mathrm{d} u_{1} \mathrm{~d} u_{2} \cdots \mathrm{d} u_{m}\right)^{p} x^{t} \mathrm{~d} x\right]^{1 / p} \leq \int_{(0, \infty)^{m}}\left(\int_{0}^{\infty} \frac{\prod_{i=1}^{m}\left|f_{i}\left(u_{i} x\right)\right|^{p}}{\prod_{i=1}^{m}\left(1+u_{i}\right)^{\gamma_{i}}} x^{t} \mathrm{~d} x\right)^{1 / p} \\
& \cdot\left(\prod_{i=1}^{m} u_{i}^{\beta_{i}}\right) \mathrm{d} u_{1} \mathrm{~d} u_{2} \cdots \mathrm{d} u_{m} \\
& =\int_{(0, \infty)^{m}} \frac{\prod_{i=1}^{m} u_{i}^{\beta_{i}}}{\prod_{i=1}^{m}\left(1+u_{i}\right)^{\gamma_{i}}}\left(\int_{0}^{\infty} \prod_{i=1}^{m}\left|f_{i}\left(u_{i} x\right)\right|^{p} x^{t} \mathrm{~d} x\right)^{1 / p} \mathrm{~d} u_{1} \mathrm{~d} u_{2} \cdots \mathrm{d} u_{m} .
\end{aligned}
$$

Using (6), (7), and Hölder's inequality, we easily obtain that

$$
\begin{aligned}
& \int_{0}^{\infty} \prod_{i=1}^{m}\left|f_{i}\left(u_{i} x\right)\right|^{p} x^{t} \mathrm{~d} x \\
& \leq \prod_{i=1}^{m}\left(\int_{0}^{\infty}\left|f_{i}\left(u_{i} x\right)\right|^{p_{i}} x^{t_{i}} \mathrm{~d} x\right)^{p / p_{i}} \\
& \quad=\prod_{i=1}^{m} u_{i}^{-\left(p / p_{i}\right)\left(t_{i}+1\right)}\left\|f_{i}\right\|_{p_{i}, t_{i}}^{p}
\end{aligned}
$$

It follows that

$$
\begin{aligned}
& \left\|T_{\vec{\beta}, \vec{\gamma}}(\vec{f})\right\|_{p, t} \leq\left(\prod_{i=1}^{m}\left\|f_{i}\right\|_{p_{i}, t_{i}}\right) \\
& \cdot \int_{(0, \infty)^{m}} \frac{\prod_{i=1}^{m} u_{i}^{\beta_{i}-\left(t_{i}+1\right) / p_{i}}}{\prod_{i=1}^{m}\left(1+u_{i}\right)^{\gamma_{i}}} \mathrm{~d} u_{1} \mathrm{~d} u_{2} \cdots \mathrm{d} u_{m}
\end{aligned}
$$

$$
\begin{aligned}
& =\left(\prod_{i=1}^{m}\left\|f_{i}\right\|_{p_{i}, t_{i}}\right) \prod_{i=1}^{m} \int_{0}^{\infty} \frac{u_{i}^{\beta_{i}-\left(t_{i}+1\right) / p_{i}}}{\left(1+u_{i}\right)^{\gamma_{i}}} \mathrm{~d} u_{i} \\
& =\left(\prod_{i=1}^{m}\left\|f_{i}\right\|_{p_{i}, t_{i}}\right) \\
& \cdot\left(\prod_{i=1}^{m} B\left(\beta_{i}+1-\frac{t_{i}+1}{p_{i}}, \gamma_{i}-\beta_{i}-1+\frac{t_{i}+1}{p_{i}}\right)\right) .
\end{aligned}
$$

The proof is complete.

We also have the following result.

Lemma 6. Let $\beta_{1}, \ldots, \beta_{m}, \gamma_{1}, \ldots, \gamma_{m}$ be real numbers. Let $1<$ $p_{1}, \ldots, p_{l}<\infty, 1 \leq l<m, 1 \leq p<\infty$, and assume that $1 / p_{1}+$ $\cdots+1 / p_{l}=1 / p$. Let $t_{1}, \ldots, t_{l}, t$ be real numbers and assume 
that $t_{1} / p_{1}+\cdots+t_{l} / p_{l}=t / p$. Suppose that the parameters satisfy

$$
\begin{gathered}
\sum_{j=1}^{m} \gamma_{j}=\sum_{j=1}^{m} \beta_{j}+m ; \\
-p_{j}\left(\gamma_{j}-\beta_{j}-1\right)<t_{j}+1<p_{j}\left(\beta_{j}+1\right), \\
\text { for } j=1, \ldots, l, \\
\gamma_{j}>\beta_{j}+1>0, \\
\text { for } j=l+1, \ldots, m .
\end{gathered}
$$

Then the operator $T_{\vec{\beta}, \vec{\gamma}}$ is bounded from $L_{t_{1}}^{p_{1}}((0, \infty)) \times \cdots \times$ $L_{t_{l}}^{p_{l}}((0, \infty)) \times\left(L^{\infty}((0, \infty))\right)^{m-l}$ to $L_{t}^{p}((0, \infty))$. In this case, if we denote by $\left\|T_{\vec{\beta}, \vec{r}}\right\|_{\left(\prod_{j=1}^{l} L_{t_{j}}^{p_{j}}\right) \times\left(L^{\infty}\right)^{m-l} \rightarrow L_{t}^{p}}$ the operator norm of $T_{\vec{\beta}, \vec{r}}$, then

$$
\begin{aligned}
& \left\|T_{\vec{\beta}, \vec{\gamma}}\right\|_{\left(\prod_{j=1}^{l} L_{t_{j}}^{p_{j}}\right) \times\left(L^{\infty}\right)^{m-l} \rightarrow L_{t}^{p}} \\
& \leq\left[\prod_{j=1}^{l} B\left(\beta_{j}+1-\frac{t_{j}+1}{p_{j}}, \gamma_{j}-\beta_{j}-1+\frac{t_{j}+1}{p_{j}}\right)\right] \\
& \quad \times\left[\prod_{j=l+1}^{m} B\left(\beta_{j}+1, \gamma_{j}-\beta_{j}-1\right)\right] .
\end{aligned}
$$

Proof. For $f_{i} \in L_{t_{i}}^{p_{i}}, i=1, \ldots, l$, and $f_{l+1}, \ldots, f_{m} \in L^{\infty}$, we write again $\vec{f}=\left(f_{1}, \ldots, f_{m}\right)$. Proceeding as at the beginning of the proof of the previous lemma, we obtain

$$
\begin{aligned}
& \left\|T_{\vec{\beta}, \vec{\gamma}}(\vec{f})\right\|_{p, t} \leq\left(\prod_{j=l+1}^{m}\left\|f_{j}\right\|_{\infty}\right) \int_{(0, \infty)^{m}} \frac{\prod_{j=1}^{m} u_{j}^{\beta_{j}}}{\prod_{j=1}^{m}\left(1+u_{j}\right)^{\gamma_{j}}}\left(\int_{0}^{\infty} \prod_{i=1}^{l}\left|f_{i}\left(u_{i} x\right)\right|^{p} x^{t} \mathrm{~d} x\right)^{1 / p} \mathrm{~d} u_{1} \mathrm{~d} u_{2} \cdots \mathrm{d} u_{m} \leq\left(\prod_{j=l+1}^{m}\left\|f_{j}\right\|_{\infty}\right) \\
& \cdot\left(\prod_{j=1}^{l}\left\|f_{j}\right\|_{p_{j}, t_{j}}\right) \int_{(0, \infty)^{m}} \frac{\left(\prod_{i=1}^{l} u_{i}^{\beta_{i}-\left(t_{i}+1\right) / p_{i}}\right)\left(\prod_{i=l+1}^{m} u_{i}^{\beta_{i}}\right)}{\prod_{i=1}^{m}\left(1+u_{i}\right)^{\gamma_{i}}} \mathrm{~d} u_{1} \mathrm{~d} u_{2} \cdots \mathrm{d} u_{m}=\left(\prod_{j=l+1}^{m}\left\|f_{j}\right\|_{\infty}\right)\left(\prod_{j=1}^{l}\left\|f_{j}\right\|_{p_{j}, t_{j}}\right) \\
& \cdot\left(\prod_{i=1}^{l} B\left(\beta_{i}+1-\frac{t_{i}+1}{p_{i}}, \gamma_{i}-\beta_{i}-1+\frac{t_{i}+1}{p_{i}}\right)\right) \times\left(\prod_{i=l+1}^{m} B\left(\beta_{i}+1, \gamma_{i}-\beta_{i}-1\right)\right) .
\end{aligned}
$$

The proof is complete.

Let us now prove the lower bound for the norm of $T_{\vec{\beta}, \vec{\gamma}}$.

Lemma 7. Let $\beta_{1}, \ldots, \beta_{m}, \gamma_{1}, \ldots, \gamma_{m}$ be real numbers. Let $1<$ $p_{1}, \ldots, p_{m} \leq \infty, 1 \leq p<\infty$, and let $t_{1}, \ldots, t_{m}$, t be real numbers and assume that relations (6) and (7) hold. Suppose that the parameters satisfy

$$
\begin{gathered}
\sum_{j=1}^{m} \gamma_{j}=\sum_{j=1}^{m} \beta_{j}+m, \\
-\left(\gamma_{j}-\beta_{j}-1\right)<\frac{t_{j}+1}{p_{j}}<\beta_{j}+1, \quad j=1, \ldots, m .
\end{gathered}
$$

Then

$$
\begin{aligned}
& \left\|T_{\vec{\beta}, \vec{\gamma}}\right\|_{\left(\prod_{j=1}^{m} L_{t_{j}}^{p_{j}}\right) \rightarrow L_{t}^{p}} \\
& \quad \geq \prod_{j=1}^{m} B\left(\beta_{j}+1-\frac{t_{j}+1}{p_{j}}, \gamma_{j}-\beta_{j}-1+\frac{t_{j}+1}{p_{j}}\right) .
\end{aligned}
$$

Proof. For simplicity, let us put

$$
\begin{aligned}
K & =\left\|T_{\vec{\beta}, \vec{\gamma}}\right\|_{\left(\prod_{j=1}^{m} L_{t_{j}}^{p_{j}}\right) \rightarrow L_{t}^{p}}, \\
B_{m} & :=\prod_{j=1}^{m} B\left(\beta_{j}+1-\frac{t_{j}+1}{p_{j}}, \gamma_{j}-\beta_{j}-1+\frac{t_{j}+1}{p_{j}}\right) .
\end{aligned}
$$

For any vector $\vec{f}=\left(f_{1}, \ldots, f_{m}\right), f_{j} \in L_{t_{j}}^{p_{j}}((0, \infty)), j=$ $1, \ldots, m$ (for $p_{j}=\infty$, replace $L_{t_{j}}^{p_{j}}((0, \infty))$ by $L^{\infty}((0, \infty))$ ), we have

$$
K\left(\prod_{j=1}^{m}\left\|f_{j}\right\|_{p_{j}, t_{j}}\right) \geq\left\|T_{\vec{\beta}, \vec{\gamma}}(\vec{f})\right\|_{p, t}
$$

hence for any $g \in L_{t}^{p^{\prime}}((0, \infty))$ (with $L_{t}^{p^{\prime}}((0, \infty))$ replaced by $L^{\infty}((0, \infty))$ if $\left.p=1\right)$,

$$
K\left(\prod_{j=1}^{m}\left\|f_{j}\right\|_{p_{j}, t_{j}}\right) \geq\left|\int_{0}^{\infty} g(x) T_{\vec{\beta}, \vec{\gamma}}(\vec{f})(x) x^{t} \mathrm{~d} x\right| .
$$

Let $0<\xi<\min \left\{p_{j}\left(\beta_{j}+1\right)-\left(t_{j}+1\right): j=1, \ldots, m\right\}$ and define

$$
\begin{aligned}
& f_{j}(x)= \begin{cases}0 & \text { if } 0<x<1 \\
x^{-\left(t_{j}+1+\xi\right) / p_{j}} & \text { if } x \geq 1,\end{cases} \\
& g(x)= \begin{cases}0 & \text { if } 0<x<1 \\
x^{-(t+1+\xi) / p^{\prime}} & \text { if } x \geq 1 .\end{cases}
\end{aligned}
$$


Then

$$
\begin{aligned}
\left\|f_{j}\right\|_{p_{j}, t_{j}} & =\frac{1}{\xi^{1 / p_{j}}}, \\
\|g\|_{p^{\prime}, t} & =\frac{1}{\xi^{1 / p^{\prime}}} .
\end{aligned}
$$

Substituting these into (36), we obtain

$$
\begin{aligned}
& \frac{K}{\xi} \geq \int_{1}^{\infty} x^{-(t+1+\xi) / p^{\prime}}\left(\int_{(1, \infty)^{m}} \frac{\prod_{j=1}^{m} y_{j}^{\beta_{j}-\left(t_{j}+1+\xi\right) / p_{j}}}{\prod_{j=1}^{m}\left(x+y_{j}\right)^{\gamma_{j}}} \mathrm{~d} y_{1}\right. \\
& \left.\quad \cdots \mathrm{d} y_{m}\right) x^{t} \mathrm{~d} x
\end{aligned}
$$

Observing that $\int_{1}^{\infty}=\int_{0}^{\infty}-\int_{0}^{1}$, we obtain that

$$
\begin{aligned}
L:= & \int_{(1, \infty)^{m}} \frac{\prod_{j=1}^{m} y_{j}^{\beta_{j}-\left(t_{j}+1+\xi\right) / p_{j}}}{\prod_{j=1}^{m}\left(x+y_{j}\right)^{\gamma_{j}}} \mathrm{~d} y_{1} \cdots \mathrm{d} y_{m} \\
= & \int_{(0, \infty)^{m}} \frac{\prod_{j=1}^{m} y_{j}^{\beta_{j}-\left(t_{j}+1+\xi\right) / p_{j}}}{\prod_{j=1}^{m}\left(x+y_{j}\right)^{\gamma_{j}}} \mathrm{~d} y_{1} \cdots \mathrm{d} y_{m} \\
& +\sum_{k, l \neq 0, k+l=m}(-1)^{l} L_{k} L_{l} \\
& +(-1)^{m} \int_{(0,1)^{m}} \frac{\prod_{j=1}^{m} y_{j}^{\beta_{j}-\left(t_{j}+1+\xi\right) / p_{j}}}{\prod_{j=1}^{m}\left(x+y_{j}\right)^{\gamma_{j}}} \mathrm{~d} y_{1} \cdots \mathrm{d} y_{m} \\
= & \left(x-\sum_{j=1}^{m}\left(t_{j}+1+\xi\right) / p_{j}\right) B_{m}+\sum_{k, l \neq 0, k+l=m}(-1)^{l} L_{k} L_{l} \\
& +(-1)^{m} \int_{(0,1)^{m}} \frac{\prod_{j=1}^{m} y_{j}^{\beta_{j}-\left(t_{j}+1+\xi\right) / p_{j}}}{\prod_{j=1}^{m}\left(x+y_{j}\right)^{\gamma_{j}}} \mathrm{~d} y_{1} \cdots \mathrm{d} y_{m},
\end{aligned}
$$

where

$$
\begin{aligned}
& L_{k}:=\int_{(0, \infty)^{k}} \frac{\prod_{s=1}^{k} y_{j_{s}}^{\beta_{j_{s}}-\left(t_{j_{s}}+1+\xi\right) / p_{j_{s}}}}{\prod_{s=1}^{k}\left(x+y_{j_{s}}\right)^{\gamma_{j_{s}}}} \mathrm{~d} y_{j_{1}} \cdots \mathrm{d} y_{j_{k}}, \\
& L_{l}:=\int_{(0,1)^{l}} \frac{\prod_{s=1}^{l} y_{j_{s}}^{\beta_{j_{s}}-\left(t_{j_{s}}+1+\xi\right) / p_{j_{s}}}}{\prod_{s=1}^{l}\left(x+y_{j_{s}}\right)^{\gamma_{j_{s}}}} \mathrm{~d} y_{j_{1}} \cdots \mathrm{d} y_{j_{k}} .
\end{aligned}
$$

We have that

$$
0<L_{k}=\left(x^{-\sum_{s=1}^{k} \gamma_{j_{s}}+\sum_{s=1}^{k} \beta_{j_{s}}+k-\sum_{s=1}^{k}\left(\left(t_{j_{s}}+1+\xi\right) / p_{j_{s}}\right)}\right) B_{k}(\xi)
$$

where

$$
\begin{aligned}
& B_{k}(\xi) \\
& :=\prod_{s=1}^{k} B\left(\beta_{j_{s}}+1-\frac{t_{j_{s}}+1+\xi}{p_{j_{s}}}, \gamma_{j_{s}}-\beta_{j_{s}}-1+\frac{t_{j_{s}}+1}{p_{j_{s}}}\right), \\
& 0<L_{l} \\
& \leq\left(x^{-\sum_{s=1}^{l} \gamma_{j_{s}}}\right) \int_{(0,1)^{l}}\left(\prod_{s=1}^{l} y_{j_{s}}^{\beta_{j_{s}}-\left(t_{j_{s}}+1+\xi\right) / p_{j_{s}}}\right) \mathrm{d} y_{j_{1}} \cdots \mathrm{d} y_{j_{k}} \\
& =\left(x^{-\sum_{s=1}^{l} \gamma_{j_{s}}}\right)\left(\prod_{s=1}^{l} \frac{1}{\beta_{j_{s}}+1-\left(t_{j_{s}}+1+\xi\right) / p_{j_{s}}}\right) \\
& :=\left(x^{-\sum_{s=1}^{l} \gamma_{j_{s}}}\right) C_{l}(\xi) .
\end{aligned}
$$

Let us write

$$
L_{0}:=\int_{(0,1)^{m}} \frac{\prod_{j=1}^{m} y_{j}^{\beta_{j}-\left(t_{j}+1+\xi\right) / p_{j}}}{\prod_{j=1}^{m}\left(x+y_{j}\right)^{\gamma_{j}}} \mathrm{~d} y_{1} \cdots \mathrm{d} y_{m} .
$$

Then

$$
0<L_{0} \leq\left(x^{-\sum_{j=1}^{m} \gamma_{j}}\right) C_{m}(\xi)
$$

with

$$
C_{m}(\xi):=\prod_{j=1}^{m} \frac{1}{\beta_{j}+1-\left(t_{j}+1+\xi\right) / p_{j}}
$$

It follows that if $m$ is an even integer, then

$$
\begin{aligned}
L> & \left(x^{-\sum_{j=1}^{m}\left(\left(t_{j}+1+\xi\right) / p_{j}\right)}\right) B_{m} \\
& -\sum_{k \neq 0, k+2 l+1=m} x^{\alpha_{k}} B_{k}(\xi) C_{2 l+1}(\xi),
\end{aligned}
$$

where $\alpha_{k}:=-\sum_{j=1}^{m} \gamma_{j}+\sum_{s=1}^{k} \beta_{j_{s}}+k-\sum_{s=1}^{k}\left(\left(t_{j_{s}}+1+\xi\right) / p_{j_{s}}\right)$; and if $m$ is an odd number, then

$$
\begin{aligned}
L> & \left(x^{-\sum_{j=1}^{m}\left(\left(t_{j}+1+\xi\right) / p_{j}\right)}\right) B_{m} \\
& -\sum_{k \neq 0, k+2 l+1=m} x^{\alpha_{k}} B_{k}(\xi) C_{2 l+1}(\xi) \\
& -x^{-\sum_{j=1}^{m} \gamma_{j}} C_{m}(\xi) .
\end{aligned}
$$


Taking (48) into (39), we obtain that if $m$ is even, then

$$
\begin{aligned}
\frac{K}{\xi} & \geq B_{m} \int_{1}^{\infty} x^{t-(t+1+\xi) / p^{\prime}-\sum_{j=1}^{m}\left(\left(t_{j}+1+\xi\right) / p_{j}\right)} \mathrm{d} x \\
& -\sum_{k \neq 0, k+2 l+1=m} B_{k}(\xi) C_{2 l+1}(\xi) \int_{1}^{\infty} x^{t-(t+1+\xi) / p^{\prime}+\alpha_{k}} \mathrm{~d} x \\
& =B_{m} \int_{1}^{\infty} x^{-1-\xi} \mathrm{d} x-\sum_{k \neq 0, k+2 l+1=m} B_{k}(\xi) C_{2 l+1}(\xi) \\
& \cdot \int_{1}^{\infty} x^{-\sum_{s=1}^{2 l+1}\left(\beta_{j_{s}}+1-\left(t_{j_{s}}+1+\xi\right) / p_{j_{s}}\right)-1-\xi} \mathrm{d} x=\frac{B_{m}}{\xi} \\
& -\sum_{k \neq 0, k+2 l+1=m} \frac{B_{k}(\xi) C_{2 l+1}(\xi)}{\sum_{s=1}^{2 l+1}\left(\beta_{j_{s}}+1-\left(t_{j_{s}}+1+\xi\right) / p_{j_{s}}\right)+\xi} .
\end{aligned}
$$

Thus

$$
\begin{aligned}
K & \geq B_{m} \\
& -\sum_{k \neq 0, k+2 l+1=m} \frac{\xi B_{k}(\xi) C_{2 l+1}(\xi)}{\sum_{s=1}^{2 l+1}\left(\beta_{j_{s}}+1-\left(t_{j_{s}}+1+\xi\right) / p_{j_{s}}\right)+\xi} .
\end{aligned}
$$

Taking (49) into (39) and doing the same type of calculations as above, we obtain that if $m$ is an odd integer, then

$$
\begin{aligned}
K & \geq B_{m} \\
& -\sum_{k \neq 0, k+2 l+1=m} \frac{\xi B_{k}(\xi) C_{2 l+1}(\xi)}{\sum_{s=1}^{2 l+1}\left(\beta_{j_{s}}+1-\left(t_{j_{s}}+1+\xi\right) / p_{j_{s}}\right)+\xi} \\
& -\xi \frac{C_{m}(\xi)}{\xi+\sum_{j=1}^{m}\left(\beta_{j}+1-\left(t_{j}+1+\xi\right) / p_{j}\right)} .
\end{aligned}
$$

$$
\begin{aligned}
& \left\|S_{\vec{\beta}, \gamma}(\vec{f})\right\|_{p, t}=\left(\int_{0}^{\infty}\left|S_{\vec{\beta}, \gamma}(\vec{f})(x)\right|^{p} x^{t} \mathrm{~d} x\right)^{1 / p} \\
& \quad \leq\left[\int_{0}^{\infty}\left(\int_{(0, \infty)^{m}} \frac{\prod_{i=1}^{m}\left|f_{i}\left(y_{i}\right)\right|}{\left(x+y_{1}+\cdots+y_{m}\right)^{\gamma}}\left(\prod_{i=1}^{m} y_{i}^{\beta_{i}}\right) \mathrm{d} y_{1} \mathrm{~d} y_{2} \cdots \mathrm{d} y_{m}\right)^{p} x^{t} \mathrm{~d} x\right]^{1 / p} \\
& \quad=\left[\int_{0}^{\infty}\left(\int_{(0, \infty)^{m}} \frac{\prod_{i=1}^{m}\left|f_{i}\left(u_{i} x\right)\right|}{\left(1+u_{1}+\cdots+u_{m}\right)^{\gamma}}\left(\prod_{i=1}^{m} u_{i}^{\beta_{i}}\right) \mathrm{d} u_{1} \mathrm{~d} u_{2} \cdots \mathrm{d} u_{m}\right)^{p} x^{t} \mathrm{~d} x\right]^{1 / p} \\
& \quad \leq \int_{(0, \infty)^{m}}\left(\int_{0}^{\infty} \frac{\prod_{i=1}^{m}\left|f_{i}\left(u_{i} x\right)\right|^{p}}{\left(1+u_{1}+\cdots+u_{m}\right)^{\gamma}} x^{t} \mathrm{~d} x\right)^{1 / p}\left(\prod_{i=1}^{m} u_{i}^{\beta_{i}}\right) \mathrm{d} u_{1} \mathrm{~d} u_{2} \cdots \mathrm{d} u_{m} \\
& \quad=\int_{(0, \infty)^{m}} \frac{\prod_{i=1}^{m} u_{i}^{\beta_{i}}}{\left(1+u_{1}+\cdots+u_{m}\right)^{\gamma}}\left(\int_{0}^{\infty} \prod_{i=1}^{m}\left|f_{i}\left(u_{i} x\right)\right|^{p} x^{t} \mathrm{~d} x\right)^{1 / p} \mathrm{~d} u_{1} \mathrm{~d} u_{2} \cdots \mathrm{d} u_{m} \leq\left(\prod_{i=1}^{m}\left\|f_{i}\right\|_{p_{i}, t_{i}}\right)
\end{aligned}
$$
and equality (23), we obtain $\left\|S_{\vec{\beta}, \gamma}^{m}\right\|_{\left(\prod_{j=1}^{m} L_{t_{j}}^{p_{j}}\right) \rightarrow L_{t}^{p_{m}}}$ the operator norm of $S_{\vec{\beta}, \gamma}$, then

The proof is complete.

$$
0<\gamma=\sum_{j=1}^{m} \beta_{j}+m
$$

$$
t>-1
$$

Proof. The proof is essentially the same as above. Let us give it here for completeness. For $f_{i} \in L_{t_{i}}^{p_{i}}, i=1, \ldots, m$, we write again $\vec{f}=\left(f_{1}, \ldots, f_{m}\right)$. We remark that $t>-1$ and $\gamma=\sum_{i=1}^{m}\left(\beta_{i}+1-\left(t_{i}+1\right) / p_{i}\right)+(t+1) / p$. Using an easy change of variables and Minkowski's inequality, Hölder's inequality,

4.2. The Norm of $S_{\vec{\beta}, \gamma}$. Let us prove the following upper bound

Then the operator $S_{\vec{\beta}, \gamma}$ is bounded from $L_{t_{1}}^{p_{1}}((0, \infty)) \times \cdots \times$ $L_{t_{m}}^{p_{m}}((0, \infty))$ to $L_{t}^{p}((0, \infty))$. In this case, if we denote by

$$
\begin{aligned}
& \left\|S_{\vec{\beta}, \gamma}\right\|_{\left(\prod_{j=1}^{m} L_{t_{j}}^{p_{j}}\right) \rightarrow L_{t}^{p}} \\
& \quad \leq \frac{\left(\prod_{j=1}^{m} \Gamma\left(\beta_{j}+1-\left(t_{j}+1\right) / p_{j}\right)\right) \Gamma((t+1) / p)}{\Gamma(\gamma)} .
\end{aligned}
$$

$$
\begin{aligned}
& \left\|T_{\vec{\beta}, \vec{\gamma}}\right\|_{\left(\prod_{j=1}^{m} L_{t_{j}}^{p_{j}}\right) \rightarrow L_{t}^{p}} \\
& \quad \geq \prod_{j=1}^{m} B\left(\beta_{j}+1-\frac{t_{j}+1}{p_{j}}, \gamma_{j}-\beta_{j}-1+\frac{t_{j}+1}{p_{j}}\right) .
\end{aligned}
$$

(1)

Letting $\xi \rightarrow 0$ in (51) or (52) we obtain that $K \geq B_{m}$. That is,
Lemma 8. Let $\beta_{1}, \ldots, \beta_{m}, \gamma$ be real numbers. Let $1<$ , $1 \leq p<\infty$, and let $t_{1}, \ldots, t_{m}, t$ be real numbers, and assume that relations (6) and (7) hold. Assume that for the norm of $S_{\vec{\beta}, \gamma}$




$$
\cdot \int_{(0, \infty)^{m}} \frac{\prod_{i=1}^{m} u_{i}^{\beta_{i}-\left(t_{i}+1\right) / p_{i}}}{\left(1+u_{1}+\cdots+u_{m}\right)^{\gamma}} \mathrm{d} u_{1} \mathrm{~d} u_{2} \cdots \mathrm{d} u_{m}=\left(\prod_{i=1}^{m}\left\|f_{i}\right\|_{p_{i}, t_{i}}\right) \frac{\left(\prod_{i=1}^{m} \Gamma\left(\beta_{i}+1-\left(t_{i}+1\right) / p_{i}\right)\right) \Gamma((t+1) / p)}{\Gamma(\gamma)} .
$$

The proof is complete.

Let us prove the following result.

Lemma 9. Let $\beta_{1}, \ldots, \beta_{m}, \gamma_{1}, \ldots, \gamma_{m}$ be real numbers. Let $1<$ $p_{1}, \ldots, p_{l}<\infty, 1 \leq l<m, 1 \leq p<\infty$, and assume that $1 / p_{1}+$ $\cdots+1 / p_{l}=1 / p$. Let $t_{1}, \ldots, t_{l}$, t be real numbers and assume that $t_{1} / p_{1}+\cdots+t_{l} / p_{l}=t / p$. Suppose that the parameters satisfy

$$
0<\gamma=\sum_{j=1}^{m} \beta_{j}+m, \quad t>-1
$$

$$
\begin{gathered}
t_{j}+1<p_{j}\left(\beta_{j}+1\right), \text { for } j=1, \ldots, l, \\
\beta_{j}>-1, \quad \text { for } j=l+1, \ldots, m .
\end{gathered}
$$

Then the operator $S_{\vec{\beta}, \gamma}$ is bounded from $L_{t_{1}}^{p_{1}}((0, \infty)) \times \cdots \times$ $L_{t_{l}}^{p_{l}}((0, \infty)) \times\left(L^{\infty}((0, \infty))\right)^{m-l}$ to $L_{t}^{p}((0, \infty))$. In this case, if we denote by $\left\|S_{\vec{\beta}, \gamma}\right\|_{\left(\prod_{j=1}^{l} L_{t_{j}}^{p_{j}}\right) \times\left(L^{\infty}\right)^{m-l} \rightarrow L_{t}^{p}}$ the operator norm of $S_{\vec{\beta}, \gamma^{\prime}}$, then

$$
\left\|S_{\vec{\beta}, \gamma}\right\|_{\left(\prod_{j=1}^{l} L_{t_{j}}^{p_{j}}\right) \times\left(L^{\infty}\right)^{m-l} \rightarrow L_{t}^{p}} \leq \frac{\left(\prod_{j=1}^{l} \Gamma\left(\beta_{j}+1-\left(t_{j}+1\right) / p_{j}\right)\right) \Gamma((t+1) / p)}{\Gamma(\gamma)}\left[\prod_{j=l+1}^{m} \Gamma\left(\beta_{j}+1\right)\right] .
$$

Proof. For $f_{i} \in L_{t_{i}}^{p_{i}}, i=1, \ldots, l$, and $f_{l+1}, \ldots, f_{m} \in L^{\infty}$, we write again $\vec{f}=\left(f_{1}, \ldots, f_{m}\right)$. We observe that $t>-1$ and $\gamma=\sum_{i=1}^{l}\left(\beta_{i}+1-\left(t_{i}+1\right) / p_{i}\right)+\sum_{i=l+1}^{m}\left(\beta_{i}+1\right)+(t+1) / p$.
We proceed as in the proof of Lemma 8; we use a change of variables and Minkowski's inequality, Hölder's inequality, and (23) to obtain

$$
\begin{aligned}
\left\|S_{\vec{\beta}, \gamma}(\vec{f})\right\|_{p, t} & \leq\left(\prod_{j=l+1}^{m}\left\|f_{j}\right\|_{\infty}\right) \int_{(0, \infty)^{m}} \frac{\prod_{i=1}^{m} u_{i}^{\beta_{i}}}{\left(1+u_{1}+\cdots+u_{m}\right)^{\gamma}} \times\left(\int_{0}^{\infty} \prod_{i=1}^{l}\left|f_{i}\left(u_{i} x\right)\right|^{p} x^{t} \mathrm{~d} x\right)^{1 / p} \mathrm{~d} u_{1} \mathrm{~d} u_{2} \cdots \mathrm{d} u_{m} \\
& \leq\left(\prod_{j=l+1}^{m}\left\|f_{j}\right\|_{\infty}\right)\left(\prod_{i=1}^{l}\left\|f_{i}\right\|_{p_{i}, t_{i}}\right) \times \int_{(0, \infty)^{m}} \frac{\left(\prod_{i=1}^{l} u_{i}^{\beta_{i}-\left(t_{i}+1\right) / p_{i}}\right)\left(\prod_{i=l+1}^{m} u_{i}^{\beta_{i}}\right)}{\left(1+u_{1}+\cdots+u_{m}\right)^{\gamma}} \mathrm{d} u_{1} \mathrm{~d} u_{2} \cdots \mathrm{d} u_{m} \\
& =\frac{\left(\prod_{i=1}^{l} \Gamma\left(\beta_{i}+1-\left(t_{i}+1\right) / p_{i}\right)\right)\left(\prod_{i=l+1}^{m} \Gamma\left(\beta_{i}+1\right)\right) \Gamma((t+1) / p)}{\Gamma(\gamma)} \times\left(\prod_{j=l+1}^{m}\left\|f_{j}\right\|_{\infty}\right)\left(\prod_{i=1}^{l}\left\|f_{i}\right\|_{p_{i}, t_{i}}\right) .
\end{aligned}
$$

The proof is complete.

We next obtain a lower bound of the norm of $S_{\vec{\beta}, \gamma}(\vec{f})$.

Lemma 10. Let $\beta_{1}, \ldots, \beta_{m}, \gamma$ be real numbers. Let $1<$ $p_{1}, \ldots, p_{m} \leq \infty, 1 \leq p<\infty$, and let $t_{1}, \ldots, t_{m}, t$ be real numbers with $t>-1$ and assume that relations (6) and (7) hold. Suppose that the parameters satisfy

$$
\begin{aligned}
0 & <\gamma=\sum_{j=1}^{m} \beta_{j}+m, \\
t & >-1, \\
-\left(\gamma-\beta_{j}-1\right) & <\frac{t_{j}+1}{p_{j}}<\beta_{j}+1, \quad j=1, \ldots, m .
\end{aligned}
$$

Then

$$
\begin{aligned}
& \left\|S_{\vec{\beta}, \gamma}\right\|_{\left(\prod_{j=1}^{m} L_{t_{j}}^{p_{j}}\right) \rightarrow L_{t}^{p}} \\
& \quad \geq \frac{\left(\prod_{j=1}^{m} \Gamma\left(\beta_{j}+1-\left(t_{j}+1\right) / p_{j}\right)\right) \Gamma((t+1) / p)}{\Gamma(\gamma)} .
\end{aligned}
$$

Proof. Let us put

$$
\begin{aligned}
K & =\left\|S_{\vec{\beta}, \gamma}\right\|_{\left(\prod_{j=1}^{m} L_{t_{j}}^{p_{j}}\right) \rightarrow L_{t}^{p}}, \\
B_{m} & :=\frac{\left(\prod_{j=1}^{m} \Gamma\left(\beta_{j}+1-\left(t_{j}+1\right) / p_{j}\right)\right) \Gamma((t+1) / p)}{\Gamma(\gamma)} .
\end{aligned}
$$


Then for any vector $\vec{f}=\left(f_{1}, \ldots, f_{m}\right), f_{j} \in L_{t_{j}}^{p_{j}}((0, \infty)), j=$ $1, \ldots, m\left(\right.$ for $p_{j}=\infty$, replace $L_{t_{j}}^{p_{j}}((0, \infty))$ by $\left.L^{\infty}((0, \infty))\right)$,

$$
K\left(\prod_{j=1}^{m}\left\|f_{j}\right\|_{p_{j}, t_{j}}\right) \geq\left\|S_{\vec{\beta}, \gamma}(\vec{f})\right\|_{p, t} ;
$$

hence for any $g \in L_{t}^{p^{\prime}}((0, \infty))$ (with $L_{t}^{p^{\prime}}((0, \infty))$ replaced by $L^{\infty}((0, \infty))$ if $\left.p=1\right)$,

$$
K\left(\prod_{j=1}^{m}\left\|f_{j}\right\|_{p_{j}, t_{j}}\right) \geq\left|\int_{0}^{\infty} g(x) S_{\vec{\beta}, \gamma}(\vec{f})(x) x^{t} \mathrm{~d} x\right| .
$$

Let $0<\xi<\min \left\{p_{j}\left(\beta_{j}+1\right)-\left(t_{j}+1\right): j=1, \ldots, m\right\}$ and define

$$
\begin{aligned}
& f_{j}(x)= \begin{cases}0 & \text { if } 0<x<1 \\
x^{-\left(t_{j}+1+\xi\right) / p_{j}} & \text { if } x \geq 1\end{cases} \\
& g(x)= \begin{cases}0 & \text { if } 0<x<1 \\
x^{-(t+1+\xi) / p^{\prime}} & \text { if } x \geq 1 .\end{cases}
\end{aligned}
$$

$$
\begin{aligned}
L_{0}(x) & :=\int_{(0,1)^{m}} \frac{\prod_{j=1}^{m} y_{j}^{\beta_{j}-\left(t_{j}+1+\xi\right) / p_{j}}}{\left(x+y_{1}+\cdots+y_{m}\right)^{\gamma}} \mathrm{d} y_{1} \cdots \mathrm{d} y_{m}, \\
L_{k, l}(x) & :=\int_{(0, \infty)^{k}} \int_{(0,1)^{l}} \frac{\left(\prod_{s=1}^{k} y_{j_{s}}^{\beta_{j_{s}}-\left(t_{j_{s}}+1+\xi\right) / p_{j_{s}}}\right)\left(\prod_{s=1}^{l} y_{i_{s}}^{\beta_{i_{s}}-\left(t_{i_{s}}+1+\xi\right) / p_{i_{s}}}\right)}{\left(x+y_{1}+\cdots+y_{m}\right)^{\gamma}} \mathrm{d} y_{j_{1}} \cdots \mathrm{d} y_{j_{k}} \mathrm{~d} y_{i_{1}} \cdots \mathrm{d} y_{i_{l}} .
\end{aligned}
$$

We have

$$
\begin{gathered}
0<L_{0}(x) \leq x^{-\gamma} \prod_{j=1}^{m} \frac{1}{\beta_{j}+1-\left(t_{j}+\xi+1\right) / p_{j}} \\
\quad:=x^{-\gamma} A_{m}, \\
L_{k, l}(x) \leq\left(\int_{(0,1)^{l}}\left(\prod_{s=1}^{l} y_{i_{s}}^{\beta_{i_{s}}-\left(t_{i_{s}}+1+\xi\right) / p_{i_{s}}}\right) \mathrm{d} y_{i_{1}} \cdots \mathrm{d} y_{i_{l}}\right) \\
\cdot \int_{(0, \infty)^{k}} \frac{\left(\prod_{s=1}^{k} y_{j_{s}}^{\beta_{j_{s}}-\left(t_{j_{s}}+1+\xi\right) / p_{j_{s}}}\right)}{\left(x+y_{j_{1}}+\cdots+y_{j_{k}}\right)^{\gamma}} \mathrm{d} y_{j_{1}} \cdots \mathrm{d} y_{j_{k}}
\end{gathered}
$$

Then

$$
\begin{aligned}
\left\|f_{j}\right\|_{p_{j}, t_{j}} & =\frac{1}{\xi^{1 / p_{j}}}, \\
\|g\|_{p^{\prime}, t} & =\frac{1}{\xi^{1 / p^{\prime}}} .
\end{aligned}
$$

Substituting these into (64), we obtain

$$
\begin{aligned}
\frac{K}{\xi} & \geq \int_{1}^{\infty} x^{-(t+1+\xi) / p^{\prime}}\left(\int_{(1, \infty)^{m}} \frac{\prod_{j=1}^{m} y_{j}^{\beta_{j}-\left(t_{j}+1+\xi\right) / p_{j}}}{\left(x+y_{1}+\cdots+y_{m}\right)^{\gamma}} \mathrm{d} y_{1}\right. \\
& \left.\cdots \mathrm{d} y_{m}\right) x^{t} \mathrm{~d} x .
\end{aligned}
$$

Put

$$
L(x):=\int_{(1, \infty)^{m}} \frac{\prod_{j=1}^{m} y_{j}^{\beta_{j}-\left(t_{j}+1+\xi\right) / p_{j}}}{\left(x+y_{1}+\cdots+y_{m}\right)^{\gamma}} \mathrm{d} y_{1} \cdots \mathrm{d} y_{m} .
$$

Then

$$
\begin{aligned}
L(x)= & \int_{(0, \infty)^{m}} \frac{\prod_{j=1}^{m} y_{j}^{\beta_{j}-\left(t_{j}+1+\xi\right) / p_{j}}}{\left(x+y_{1}+\cdots+y_{m}\right)^{\gamma}} \mathrm{d} y_{1} \cdots \mathrm{d} y_{m} \\
& +\sum_{k, l \neq 0, k+l=m}(-1)^{l} L_{k, l}(x)+(-1)^{m} L_{0}(x),
\end{aligned}
$$

where 


$$
\begin{aligned}
A_{l} & :=\prod_{s=1}^{l} \frac{1}{\beta_{i_{s}}+1-\left(t_{i_{s}}+\xi+1\right) / p_{i_{s}}}, \\
B_{k, l}: & =\frac{\left(\prod_{s=1}^{k} \Gamma\left(\beta_{j_{s}}+1-\left(t_{j_{s}}+\xi+1\right) / p_{j_{s}}\right)\right) \Gamma\left((t+1+\xi) / p+\sum_{s=1}^{l}\left(\beta_{i_{s}}+1-\left(t_{i_{s}}+\xi+1\right) / p_{i_{s}}\right)\right)}{\Gamma(\gamma)} .
\end{aligned}
$$

It follows that, for $m$ even,

$$
\begin{aligned}
& L(x)>x^{-\sum_{j=1}^{m}\left(t_{j}+1+\xi\right) / p_{j}} B_{m} \\
& -\sum_{k \neq 0, k+2 l+1=m}\left(x^{-\gamma+\sum_{s=1}^{k}\left(\beta_{j_{s}}+1-\left(t_{j_{s}}+1+\xi\right) / p_{j_{s}}\right)}\right) \\
& \cdot A_{2 l+1} B_{k, 2 l+1}
\end{aligned}
$$

and, for $m$ odd,

$$
\begin{aligned}
& L(x)>x^{-\sum_{j=1}^{m}\left(t_{j}+1+\xi\right) / p_{j}} B_{m} \\
& -\sum_{k \neq 0, k+2 l+1=m}\left(x^{-\gamma+\sum_{s=1}^{k}\left(\beta_{j_{s}}+1-\left(t_{j_{s}}+1+\xi\right) / p_{j_{s}}\right)}\right) \\
& \cdot A_{2 l+1} B_{k, 2 l+1}-x^{-\gamma} A_{m} .
\end{aligned}
$$

Taking (73) into (67), we obtain that if $m$ is even, then

$$
\begin{aligned}
\frac{K}{\xi} & \geq B_{m} \int_{1}^{\infty} x^{-1-\xi} \mathrm{d} x-\sum_{k \neq 0, k+2 l+1=m} A_{2 l+1} B_{k, 2 l+1} \\
& \cdot \int_{1}^{\infty} x^{-1-\xi-\sum_{s=1}^{l} \beta_{i_{s}}+1-\left(t_{i_{s}}+\xi+1\right) / p_{i_{s}}} \mathrm{~d} x=\frac{B_{m}}{\xi} \\
& -\sum_{k \neq 0, k+2 l+1=m} A_{2 l+1} B_{k, 2 l+1} \\
& \frac{1}{\xi+\sum_{s=1}^{l} \beta_{i_{s}}+1-\left(t_{i_{s}}+\xi+1\right) / p_{i_{s}}}
\end{aligned}
$$

Thus

$$
\begin{gathered}
K \geq B_{m}-\sum_{k \neq 0, k+2 l+1=m} A_{2 l+1} B_{k, 2 l+1} \\
\cdot \frac{\xi}{\xi+\sum_{s=1}^{l} \beta_{i_{s}}+1-\left(t_{i_{s}}+\xi+1\right) / p_{i_{s}}} .
\end{gathered}
$$

Taking (74) into (67), we obtain that if $m$ is odd, then

$$
\begin{aligned}
\frac{K}{\xi} & \geq B_{m} \int_{1}^{\infty} x^{-1-\xi} \mathrm{d} x-A_{m} \int_{1}^{\infty} x^{-\gamma-1-\xi+(t+1+\xi) / p} \mathrm{~d} x \\
& -\sum_{k \neq 0, k+2 l+1=m} A_{2 l+1} B_{k, 2 l+1} \\
& \cdot \int_{1}^{\infty} x^{-1-\xi-\sum_{s=1}^{l} \beta_{i_{s}}+1-\left(t_{i_{s}}+\xi+1\right) / p_{i_{s}}} \mathrm{~d} x=\frac{B_{m}}{\xi} \\
& -\frac{A_{m}}{\gamma+\xi+(t+1+\xi) / p}-\sum_{k \neq 0, k+2 l+1=m} A_{2 l+1} B_{k, 2 l+1} \\
& \cdot \frac{1}{\xi+\sum_{s=1}^{l}\left(\beta_{i_{s}}+1-\left(t_{i_{s}}+\xi+1\right) / p_{i_{s}}\right)} .
\end{aligned}
$$

Thus for $m$ odd,

$$
\begin{aligned}
K & \geq B_{m}-\frac{\xi A_{m}}{\gamma+\xi+(t+1+\xi) / p} \\
& -\sum_{k \neq 0, k+2 l+1=m} A_{2 l+1} B_{k, 2 l+1} \\
& \cdot \frac{\xi}{\xi+\sum_{s=1}^{l}\left(\beta_{i_{s}}+1-\left(t_{i_{s}}+\xi+1\right) / p_{i_{s}}\right)} .
\end{aligned}
$$

Letting $\xi \rightarrow 0$ in (76) or (78) we obtain that $K \geq B_{m}$; that is,

$$
\begin{aligned}
& \left\|S_{\vec{\beta}, \gamma}\right\|_{\left(\prod_{j=1}^{m} L_{t_{j}}^{p_{j}}\right) \rightarrow L_{t}^{p}} \\
& \quad \geq \frac{\left(\prod_{j=1}^{m} \Gamma\left(\beta_{j}+1-\left(t_{j}+1\right) / p_{j}\right)\right) \Gamma((t+1) / p)}{\Gamma(\gamma)} .
\end{aligned}
$$

The proof is complete.

\section{Necessity for Boundedness of $T_{\vec{\beta}, \vec{\gamma}}$ and $S_{\vec{\beta}, \gamma}$}

5.1. Necessity for Boundedness of $T_{\vec{\beta}, \vec{\gamma}}$

Lemma 11. Let $\beta_{1}, \ldots, \beta_{m}, \gamma_{1}, \ldots, \gamma_{m}$ be real numbers. Let $1<$ $p_{1}, \ldots, p_{m} \leq \infty, 1 \leq p<\infty$, and let $t_{1}, \ldots, t_{m}, t$ be real numbers and assume that relations (6) and (7) hold. If the operator $T_{\vec{\beta}, \vec{\gamma}}$ is bounded from $L_{t_{1}}^{p_{1}}((0, \infty)) \times \cdots \times L_{t_{m}}^{p_{m}}((0, \infty))$ to $L_{t}^{p}((0, \infty))$, then the parameters satisfy

$$
\sum_{j=1}^{m} \gamma_{j}=\sum_{j=1}^{m} \beta_{j}+m .
$$

Proof. For simplicity, we put $\gamma=\sum_{j=1}^{m} \gamma_{j}$ and $\beta=\sum_{j=1}^{m} \beta_{j}$. Let $R>0$ be a real number. Given a function $f$, we denote, by $f_{R}$, the function defined by $f_{R}(x)=f(R x)$. One easily checks that, for $f \in L_{t_{j}}^{p_{j}}$,

$$
\left\|\left(f_{j}\right)_{R}\right\|_{p_{j}, t_{j}}=R^{-\left(t_{j}+1\right) / p_{j}}\left\|f_{j}\right\|_{p_{j}, t_{j}} .
$$

For a vector $\vec{f}=\left(f_{1}, \ldots, f_{m}\right)$, we write $\vec{f}_{R}=\left(\left(f_{1}\right)_{R}, \ldots\right.$, $\left.\left(f_{m}\right)_{R}\right)$. Using some easy changes of variables, we obtain

$$
T_{\vec{\beta}, \vec{\gamma}}\left(\vec{f}_{R}\right)(x)=R^{\gamma-\beta-m} T_{\vec{\beta}, \vec{\gamma}}(\vec{f})(R x) .
$$

It comes that

$$
\left\|T_{\vec{\beta}, \vec{\gamma}}\left(\vec{f}_{R}\right)\right\|_{p, t}=\left(R^{\gamma-\beta-m-(t+1) / p}\right)\left\|T_{\vec{\beta}, \vec{\gamma}}(\vec{f})\right\|_{p, t} .
$$


Recall that the boundedness of $T_{\vec{\beta}, \vec{\gamma}}$ means that there exists a constant $C>0$ such that, for any $f_{j} \in L_{t_{j}}^{p_{j}}, j=1, \ldots, m$,

$$
\left\|T_{\vec{\beta}, \vec{\gamma}}\left(\vec{f}_{R}\right)\right\|_{p, t} \leq C \prod_{j=1}^{m}\left\|\left(f_{j}\right)_{R}\right\|_{p_{j}, t_{j}}
$$

It follows using (81) and (83) that

$$
\begin{aligned}
& \left(R^{\gamma-\beta-m-(t+1) / p}\right)\left\|T_{\vec{\beta}, \vec{\gamma}}(\vec{f})\right\|_{p, t} \\
& \quad \leq C\left(R^{-\sum_{j=1}^{m}\left(\left(t_{j}+1\right) / p_{j}\right)}\right) \prod_{j=1}^{m}\left\|f_{j}\right\|_{p_{j}, t_{j}}
\end{aligned}
$$

or equivalently,

$$
\begin{aligned}
& \left(R^{\gamma-\beta-m-(t+1) / p+\sum_{j=1}^{m}\left(\left(t_{j}+1\right) / p_{j}\right)}\right)\left\|T_{\vec{\beta}, \vec{\gamma}}(\vec{f})\right\|_{p, t} \\
& \quad \leq C \prod_{j=1}^{m}\left\|f_{j}\right\|_{p_{j}, t_{j}} \cdot
\end{aligned}
$$

As (86) holds for any $f_{1} \in L_{t_{1}}^{p_{1}}, \ldots, f_{m} \in L_{t_{m}}^{p_{m}}$ and any real number $R>0$, we necessarily have that

$$
\gamma-\beta-m-\frac{t+1}{p}+\sum_{j=1}^{m} \frac{t_{j}+1}{p_{j}}=0
$$

which combined with (7) gives that

$$
\sum_{j=1}^{m} \gamma_{j}=\sum_{j=1}^{m} \beta_{j}+m
$$

Lemma 12. Let $\beta_{1}, \ldots, \beta_{m}, \gamma_{1}, \ldots, \gamma_{m}$ be real numbers. Let $1<$ $p_{1}, \ldots, p_{m}<\infty, 1<p<\infty$, and let $t_{1}, \ldots, t_{m}$, $t$ be real numbers and assume that relations (6) and (7) hold. Then if the operator $T_{\vec{\beta}, \vec{\gamma}}$ is bounded from $L_{t_{1}}^{p_{1}}((0, \infty)) \times \cdots \times L_{t_{m}}^{p_{m}}((0, \infty))$ to $L_{t}^{p}((0, \infty))$, then the parameters satisfy

$$
-p_{j}\left(\gamma_{j}-\beta_{j}-1\right)<t_{j}+1<p_{j}\left(\beta_{j}+1\right)
$$

$$
j=1, \ldots, m \text {. }
$$

Proof. Assume that $T_{\vec{\beta}, \vec{\gamma}}$ is bounded from $L_{t_{1}}^{p_{1}}((0, \infty)) \times \cdots \times$ $L_{t_{m}}^{p_{m}}((0, \infty))$ to $L_{t}^{p}((0, \infty))$. Then its $j$-th adjoint $T_{\vec{\beta}, \vec{\gamma}}^{*, j}$ defined by

$$
\begin{aligned}
& T_{\vec{\beta}, \vec{\gamma}}^{*, j}\left(\vec{f}_{j}\right)\left(y_{j}\right) \\
& =y_{j}^{\beta_{j}-t_{j}} \int_{(0, \infty)^{m}} \frac{g(x) \prod_{1=k \neq j}^{m} f_{k}\left(y_{k}\right)}{\prod_{k=1}^{m}\left(x+y_{k}\right)^{\gamma_{k}}} x^{t}\left(\prod_{1=k \neq j}^{m} y_{k}^{\beta_{k}} \mathrm{~d} y_{k}\right) \mathrm{d} x,
\end{aligned}
$$

where $\vec{f}_{j}=\left(f_{1}, \ldots, f_{j-1}, g, f_{j+1}, \ldots, f_{m}\right)$, is bounded from $L_{t_{1}}^{p_{1}}((0, \infty)) \times \cdots \times L_{t_{j-1}}^{p_{j-1}}((0, \infty)) \times L_{t}^{p^{\prime}}((0, \infty)) \times L_{t_{j+1}}^{p_{j+1}}((0, \infty)) \times$ $\cdots \times L_{t_{m}}^{p_{m}}((0, \infty))$ to $L_{t_{j}}^{p_{j}^{\prime}}((0, \infty))$. Let us take $g(x)=\chi_{[1,2]}(x)$ and $f_{k}(y)=\chi_{[1,2]}(y), j \neq k \in\{1, \ldots, m\}$. We observe that, for $x, y_{k} \in[1,2],\left(x+y_{k}\right)^{\gamma_{k}} \simeq 1$ while $\left(x+y_{j}\right)^{\gamma_{j}} \simeq\left(1+y_{j}\right)^{\gamma_{j}}$. It follows for this choice of functions that, for any $y_{j} \in(0, \infty)$,

$$
T_{\vec{\beta}, \vec{\gamma}}^{*, j}\left(\vec{f}_{j}\right)\left(y_{j}\right) \simeq \frac{y_{j}^{\beta_{j}-t_{j}}}{\left(1+y_{j}\right)^{\gamma_{j}}} .
$$

It follows from the boundedness of $T_{\vec{\beta}, \vec{\gamma}}^{*, j}$ that there is a constant $C>0$ such that

$$
\int_{0}^{\infty} \frac{y_{j}^{p_{j}^{\prime}\left(\beta_{j}-t_{j}\right)}}{\left(1+y_{j}\right)^{\gamma_{j} p_{j}^{\prime}}} y_{j}^{t_{j}} \mathrm{~d} y_{j} \simeq\left\|T_{\vec{\beta}, \vec{\gamma}}^{*, j}\left(\vec{f}_{j}\right)\right\|_{p_{j}^{\prime}, t_{j}} \leq C .
$$

From the properties of the Beta-function, we know that this implies that $p_{j}^{\prime}\left(\beta_{j}-t_{j}\right)+t_{j}+1>0$ and $\gamma_{j} p_{j}^{\prime}-p_{j}^{\prime}\left(\beta_{j}-t_{j}\right)-t_{j}-1>$ 0 . These two inequalities are equivalent to

$$
-p_{j}\left(\gamma_{j}-\beta_{j}-1\right)<t_{j}+1<p_{j}\left(\beta_{j}+1\right) .
$$

The proof is complete.

The proof of the following result follows the same steps as in the proof of the above lemma.

Lemma 13. Let $\beta_{1}, \ldots, \beta_{m}, \gamma_{1}, \ldots, \gamma_{m}$ be real numbers. Let $1<p_{1}, \ldots, p_{m}<\infty$, and let $t_{1}, \ldots, t_{m}, t$ be real numbers and assume that relations (6) and (7) hold with $p=1$. If the operator $T_{\vec{\beta}, \vec{\gamma}}$ is bounded from $L_{t_{1}}^{p_{1}}((0, \infty)) \times \cdots \times L_{t_{m}}^{p_{m}}((0, \infty))$ to $L_{t}^{1}((0, \infty))$, then the parameters satisfy

$$
\begin{aligned}
-p_{j}\left(\gamma_{j}-\beta_{j}-1\right)<t_{j}+1<p_{j}\left(\beta_{j}+1\right) & \\
& \\
& j=1, \ldots, m .
\end{aligned}
$$

\subsection{Necessity for Boundedness of $S_{\vec{\beta}, \gamma}$}

Lemma 14. Let $\beta_{1}, \ldots, \beta_{m}, \gamma_{1}, \ldots, \gamma_{m}$ be real numbers. Let $1<p_{1}, \ldots, p_{m} \leq \infty, 1 \leq p<\infty$, and let $t_{1}, \ldots, t_{m}, t$ be real numbers and assume that relations (6) and (7) hold. If the operator $S_{\vec{\beta}, \gamma}$ is bounded from $L_{t_{1}}^{p_{1}}((0, \infty)) \times \cdots \times L_{t_{m}}^{p_{m}}((0, \infty))$ to $L_{t}^{p}((0, \infty))$, then the parameters satisfy

$$
\gamma=\sum_{j=1}^{m} \beta_{j}+m \text {. }
$$

Proof. This is obtained exactly the same way as in the proof of Lemma 11. We leave it to the interested reader.

Lemma 15. Let $\beta_{1}, \ldots, \beta_{m}, \gamma_{1}, \ldots, \gamma_{m}$ be real numbers. Let $1<$ $p_{1}, \ldots, p_{m} \leq \infty, 1 \leq p<\infty$, and let $t_{1}, \ldots, t_{m}, t$ be real numbers and assume that relations (6) and (7) hold. If the operator $S_{\vec{\beta}, \gamma}$ is bounded from $L_{t_{1}}^{p_{1}}((0, \infty)) \times \cdots \times L_{t_{m}}^{p_{m}}((0, \infty))$ to $L_{t}^{p}((0, \infty))$, then $t>-1$ and $\gamma>0$. 
Proof. Let us take $f_{k}(y)=\chi_{[1,2]}(y), k \in\{1, \ldots, m\}$. We observe that, for $y_{1}, \ldots, y_{m} \in[1,2],\left(x+y_{1}+\cdots+y_{m}\right)^{\gamma} \simeq$ $(1+x)^{\gamma}$. It follows for this choice of functions that, for any $x \in(0, \infty)$,

$$
S_{\vec{\beta}, \gamma}(\vec{f})(x) \simeq \frac{1}{(1+x)^{\gamma}} .
$$

It follows from the boundedness of $S_{\vec{\beta}, \gamma}$ that there is a constant $C>0$ such that

$$
\int_{0}^{\infty} \frac{x^{t}}{(1+x)^{\gamma p}} \mathrm{~d} x \simeq\left\|S_{\vec{\beta}, \gamma}(\vec{f})\right\|_{p, t} \leq C .
$$

Clearly, this is only possible if $t>-1$ and $\gamma>0$. The proof is complete.
Lemma 16. Let $\beta_{1}, \ldots, \beta_{m}, \gamma_{1}, \ldots, \gamma_{m}$ be real numbers. Let $1<p_{1}, \ldots, p_{m}<\infty, 1<p<\infty$, and let $t_{1}, \ldots, t_{m}, t$ be real numbers and assume that relations (6) and (7) hold. If the operator $S_{\vec{\beta}, \gamma}$ is bounded from $L_{t_{1}}^{p_{1}}((0, \infty)) \times \cdots \times L_{t_{m}}^{p_{m}}((0, \infty))$ to $L_{t}^{p}((0, \infty))$, then the parameters satisfy

$$
\begin{aligned}
&-p_{j}\left(\gamma-\beta_{j}-1\right)<t_{j}+1<p_{j}\left(\beta_{j}+1\right) \\
& \\
& j=1, \ldots, m .
\end{aligned}
$$

Proof. Assume that $S_{\vec{\beta}, \gamma}$ is bounded from $L_{t_{1}}^{p_{1}}((0, \infty)) \times \cdots \times$ $L_{t_{m}}^{p_{m}}((0, \infty))$ to $L_{t}^{p}((0, \infty))$, Then its $j$-th adjoint $S_{\vec{\beta}, \gamma}^{*, j}$ is defined by

$$
S_{\vec{\beta}, \gamma}^{*, j}\left(\vec{f}_{j}\right)\left(y_{j}\right)=\int_{(0, \infty)^{m}} \frac{y_{j}^{\beta_{j}-t_{j}} g(x) x^{t}}{\left(x+\sum_{i=1}^{m} y_{i}\right)^{\gamma}}\left(\prod_{1=k \neq j}^{m} y_{k}^{\beta_{k}} f_{k}\left(y_{k}\right)\right) \mathrm{d} y_{1} \cdots \mathrm{d} y_{j-1} \mathrm{~d} y_{j+1} \cdots \mathrm{d} y_{m} \mathrm{~d} x,
$$

where again $\vec{f}_{j}=\left(f_{1}, \ldots, f_{j-1}, g, f_{j+1}, \ldots, f_{m}\right)$ is bounded from $L_{t_{1}}^{p_{1}}((0, \infty)) \times \cdots \times L_{t_{j-1}}^{p_{j-1}}((0, \infty)) \times L_{t}^{p^{\prime}}((0, \infty)) \times$ $L_{t_{j+1}}^{p_{j+1}}((0, \infty)) \times \cdots \times L_{t_{m}}^{p_{m}}((0, \infty))$ to $L_{t_{j}}^{p_{j}^{\prime}}((0, \infty))$. Let us take $g(x)=\chi_{[1,2]}(x)$ and $f_{k}(y)=\chi_{[1,2]}(y), j \neq k \in\{1, \ldots, m\}$. We observe that, for $x, y_{k} \in[1,2], j \neq k \in\{1, \ldots, m\}$, $\left(x+y_{1}+\cdots+y_{m}\right)^{\gamma} \simeq\left(1+y_{j}\right)^{\gamma}$. It follows for this choice of functions that, for any $y_{j} \in(0, \infty)$,

$$
S_{\vec{\beta}, \gamma}^{*, j}\left(\vec{f}_{j}\right)\left(y_{j}\right) \simeq \frac{y_{j}^{\beta_{j}-t_{j}}}{\left(1+y_{j}\right)^{\gamma}} .
$$

It follows from the boundedness of $S_{\vec{\beta}, \gamma}^{*, j}$ that there is a constant $C>0$ such that

$$
\int_{0}^{\infty} \frac{y_{j}^{p_{j}^{\prime}\left(\beta_{j}-t_{j}\right)}}{\left(1+y_{j}\right)^{\gamma p_{j}^{\prime}}} y_{j}^{t_{j}} \mathrm{~d} y_{j} \simeq\left\|S_{\vec{\beta}, \gamma}^{*, j}\left(\vec{f}_{j}\right)\right\|_{p_{j}^{\prime}, t_{j}} \leq C .
$$

This as in the proof of Lemma 12 implies that

$$
-p_{j}\left(\gamma-\beta_{j}-1\right)<t_{j}+1<p_{j}\left(\beta_{j}+1\right) .
$$

The proof is complete.

The following is obtained as above.

Lemma 17. Let $\beta_{1}, \ldots, \beta_{m}, \gamma_{1}, \ldots, \gamma_{m}$ be real numbers. Let $1<p_{1}, \ldots, p_{m}<\infty$, and let $t_{1}, \ldots, t_{m}, t$ be real numbers and assume that relations (6) and (7) hold with $p=1$. If the operator $S_{\vec{\beta}, \gamma}$ is bounded from $L_{t_{1}}^{p_{1}}((0, \infty)) \times \cdots \times L_{t_{m}}^{p_{m}}((0, \infty))$ to $L_{t}^{1}((0, \infty))$, then the parameters satisfy and

$$
-p_{j}\left(\gamma-\beta_{j}-1\right)<t_{j}+1<p_{j}\left(\beta_{j}+1\right),
$$

5.3. Necessity for the Other Cases. Let us start by proving the following

Lemma 18. Let $\beta_{1}, \ldots, \beta_{m}, \gamma_{1}, \ldots, \gamma_{m}$ be real numbers. Let $1<$ $p_{1}, \ldots, p_{l}<\infty, 1 \leq l<m, 1 \leq p<\infty$, and assume that $1 / p_{1}+\cdots+1 / p_{l}=1 / p$. Let $t_{1}, \ldots, t_{l}, t$ be real numbers and assume that $t_{1} / p_{1}+\cdots+t_{l} / p_{l}=t / p$. If the operator $T_{\vec{\beta}, \vec{\gamma}}$ is bounded from $L_{t_{1}}^{p_{1}}((0, \infty)) \times \cdots \times L_{t_{l}}^{p_{l}}((0, \infty)) \times\left(L^{\infty}((0, \infty))\right)^{m-l}$ to $L_{t}^{p}((0, \infty))$, then the parameters satisfy

$$
\begin{array}{r}
-p_{j}\left(\gamma_{j}-\beta_{j}-1\right)<t_{j}+1<p_{j}\left(\beta_{j}+1\right) \\
j=1, \ldots, l, \\
\gamma_{j}>\beta_{j}+1>0, \quad j=l+1, \ldots, m .
\end{array}
$$

Proof. Suppose that $T_{\vec{\beta}, \vec{\gamma}}$ is bounded from $L_{t_{1}}^{p_{1}}((0, \infty)) \times \cdots \times$ $L_{t_{l}}^{p_{l}}((0, \infty)) \times L^{\infty}((0, \infty))^{m-l}$ to $L_{t}^{p}((0, \infty))$. Then for $1 \leq j \leq l$, the $j$-th adjoint, $T_{\vec{\beta}, \vec{\gamma}}^{* j}$, of $T_{\vec{\beta}, \vec{\gamma}}$ is defined as in the proof of Lemmas 12 and 13 . Hence the proof of the first inequalities follows from the proof of Lemmas 12 and 13. To prove the second inequalities, we observe that, for $j>l, j$-th adjoint, $T_{\vec{\beta}, \vec{\gamma}}^{* j}$ of $T_{\vec{\beta}, \vec{\gamma}}$ is defined by

$$
\begin{aligned}
& T_{\vec{\beta}, \vec{\gamma}}^{*, j}\left(\vec{f}_{j}\right)\left(y_{j}\right) \\
& =y_{j}^{\beta_{j}} \int_{(0, \infty)^{m}} \frac{g(x) \prod_{1=k \neq j}^{m} f_{k}\left(y_{k}\right)}{\prod_{k=1}^{m}\left(x+y_{k}\right)^{\gamma_{k}}}\left(\prod_{1=k \neq j}^{m} y_{k}^{\beta_{k}} \mathrm{~d} y_{k}\right) \\
& \quad \cdot x^{t} \mathrm{~d} x,
\end{aligned}
$$


where $\vec{f}_{j}=\left(f_{1}, \ldots, f_{j-1}, g, f_{j+1}, \ldots, f_{m}\right)$ is bounded from

$$
\begin{aligned}
& \left(\prod_{k=1}^{l} L_{t_{k}}^{p_{k}}((0, \infty))\right) \times\left(L^{\infty}((0, \infty))\right)^{j-l-1} \\
& \quad \times L_{t}^{p^{\prime}}((0, \infty)) \times\left(L^{\infty}((0, \infty))\right)^{m-j}
\end{aligned}
$$

to $L^{1}((0, \infty))$. Hence the second inequality can now be established similarly as in the proof of Lemma 12 .

Let us finish with the following.

Lemma 19. Let $\beta_{1}, \ldots, \beta_{m}, \gamma_{1}, \ldots, \gamma_{m}$ be real numbers. Let $1<$ $p_{1}, \ldots, p_{l}<\infty, 1 \leq l<m, 1 \leq p<\infty$, and assume that $1 / p_{1}+\cdots+1 / p_{l}=1 / p$. Let $t_{1}, \ldots, t_{l}, t$ be real numbers and assume that $t_{1} / p_{1}+\cdots+t_{l} / p_{l}=t / p$. If the operator $S_{\vec{\beta}, \gamma}$ is bounded from $L_{t_{1}}^{p_{1}}((0, \infty)) \times \cdots \times L_{t_{l}}^{p_{l}}((0, \infty)) \times\left(L^{\infty}((0, \infty))\right)^{m-l}$ to $L_{t}^{p}((0, \infty))$, then the parameters satisfy

$$
\begin{gathered}
t_{j}+1<p_{j}\left(\beta_{j}+1\right), \quad j=1, \ldots, m, \\
\beta_{j}>-1, \quad j=l+1, \ldots, m .
\end{gathered}
$$

Proof. Suppose that $S_{\vec{\beta}, \gamma}$ is bounded from $L_{t_{1}}^{p_{1}}((0, \infty)) \times \cdots \times$ $L_{t_{l}}^{p_{l}}((0, \infty)) \times L^{\infty}((0, \infty))^{m-l}$ to $L_{t}^{p}((0, \infty))$. Then for $1 \leq j \leq l$, the $j$-th adjoint, $S_{\vec{\beta}, \gamma}^{* j}$, of $S_{\vec{\beta}, \gamma}$ is defined as in the proof of Lemmas 16 and 17. Hence the proof of the first inequality follows from the proof of Lemmas 16 and 17. To prove the second inequality, we observe that, for $j>l, S_{\vec{\beta}, \gamma}^{* j}$, of $S_{\vec{\beta}, \gamma}$, is given by

$$
S_{\vec{\beta}, \gamma}^{*, j}\left(\vec{f}_{j}\right)\left(y_{j}\right)=y_{j}^{\beta_{j}} \int_{(0, \infty)^{m}} \frac{x^{t} g(x)}{\left(x+\sum_{i=1}^{m} y_{i}\right)^{\gamma}}\left(\prod_{1=k \neq j}^{m} y_{k}^{\beta_{k}} f_{k}\left(y_{k}\right)\right) \mathrm{d} y_{1} \cdots \mathrm{d} y_{j-1} \mathrm{~d} y_{j+1} \cdots \mathrm{d} y_{m} \mathrm{~d} x,
$$

where again $\vec{f}_{j}=\left(f_{1}, \ldots, f_{j-1}, g, f_{j+1}, \ldots, f_{m}\right)$ is bounded from

$$
\begin{aligned}
& \left(\prod_{k=1}^{l} L_{t_{k}}^{p_{k}}((0, \infty))\right) \times\left(L^{\infty}((0, \infty))\right)^{j-l-1} \\
& \quad \times L_{t}^{p^{\prime}}((0, \infty)) \times\left(L^{\infty}((0, \infty))\right)^{m-j}
\end{aligned}
$$

to $L^{1}((0, \infty))$. Hence the second inequality can now be established similarly as in the proof of Lemma 16.

\section{Proof of the Results}

Theorem 1 follows from Lemmas 5, 7, 11, 12, and 13 . Theorem 2 follows from Lemmas $8,10,14,15,16$, and 17. Theorem 3 follows from Lemmas 6, 7, 11, and 18. Theorem 4 follows from Lemmas 9, 10, 14, 15, and 19. The proof is complete.

\section{Competing Interests}

The authors declare that there is no conflict of interests regarding the publication of this paper.

\section{References}

[1] G. H. Hardy, "Note on a theorem of Hilbert concerning series of positive terms," Proceedings of the London Mathematical Society, vol. 23, no. 2, pp. 45-46, 1925.

[2] G. H. Hardy, J. E. Littlewood, and G. Polya, Inequalities, Cambridge University Press, Cambridge, UK, 1952.

[3] D. S. Mitrinović, J. E. Pečarić, and A. M. Fink, Inequalities Involving Functions and Their Integrals and Derivatives, vol. 53 of Mathematics and Its Applications, Kluwer Academic Publishers, Boston, Mass, USA, 1991.
[4] B. C. Yang, "On a general Hardy-Hilbert's inequality with a best value," Chinese Annals of Mathematics, vol. 21, no. 4, pp. 401$408,2000$.

[5] Y. Bicheng, “On Hardy-Hilbert's Integral Inequality," Journal of Mathematical Analysis and Applications, vol. 261, no. 1, pp. 295306, 2001.

[6] B. Yang, "On the extended Hilbert's integral inequality," JIPAM. Journal of Inequalities in Pure and Applied Mathematics, vol. 5, no. 4, article 96, 2004.

[7] J. Bansah and B. F. Sehba, "Boundedness of a family of Hilbert-type operators and of its Bergman-type analogue," https://arxiv.org/abs/1512.02537.

[8] D. Bekollé, A. Bonami, M. M. Peloso, and F. Ricci, "Boundedness of Bergman projections on tube domains over light cones," Mathematische Zeitschrift, vol. 237, no. 1, pp. 31-59, 2001.

[9] Á. Bényi and C. Oh, "Best constants for certain multilinear integral operators," Journal of Inequalities and Applications, vol. 2006, Article ID 28582, 12 pages, 2006.

[10] B. Yang and T. M. Rassias, "On the way of weight coefficient and research for the Hilbert-type inequalities," Mathematical Inequalities \& Applications, vol. 6, no. 4, pp. 625-658, 2003.

[11] Y. Bicheng, I. Brnetić, M. Krnić, and J. Pečarić, "Generalization of HILbert and HARdy-HILbert integral inequalities," Mathematical Inequalities \&amp; Applications, vol. 8, no. 2, pp. 259272, 2005.

[12] Z. Fu, L. Grafakos, S. Lu, and F. Zhao, "Sharp bounds for $m$-linear Hardy and HILbert operators," Houston Journal of Mathematics, vol. 38, no. 1, pp. 225-244, 2012.

[13] B. C. Yang, "A multiple Hardy-Hilbert's integral inequality," Chinese Annals of Mathematics A, vol. 24, no. 6, pp. 743-750, 2003.

[14] B. Yang, "On a new multiple extension of Hilbert's integral inequality," Journal of Inequalities in Pure and Applied Mathematics, vol. 6, no. 2, article no. 39, 8 pages, 2005. 


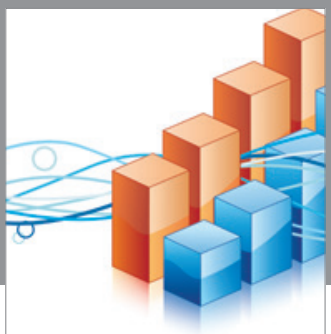

Advances in

Operations Research

vatem alat4

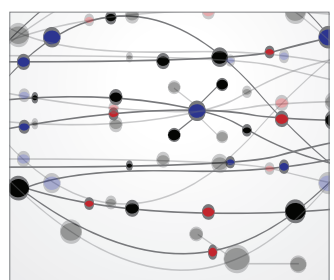

\section{The Scientific} World Journal
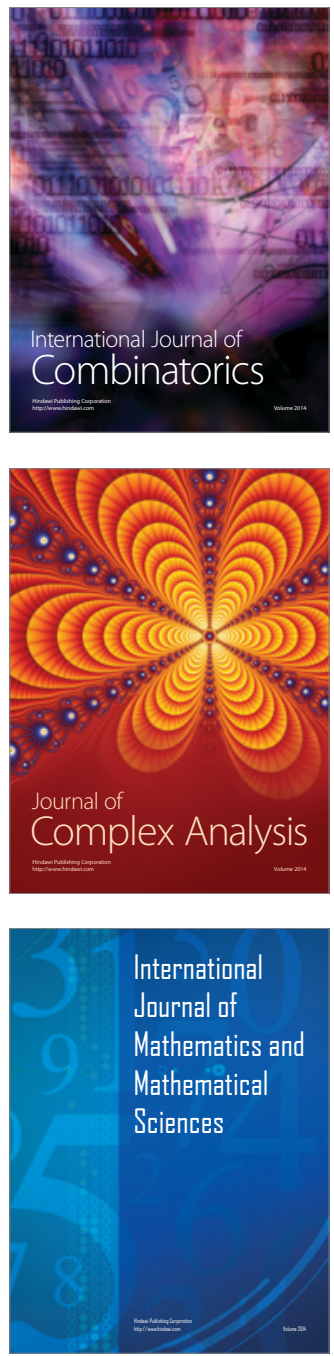
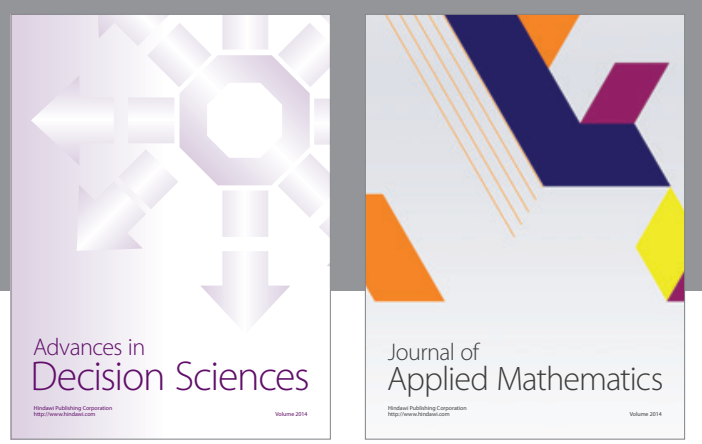

Algebra

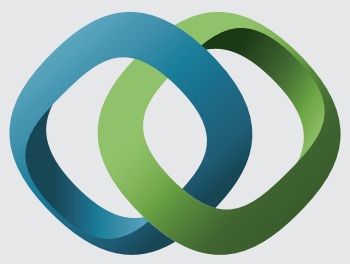

\section{Hindawi}

Submit your manuscripts at

https://www.hindawi.com
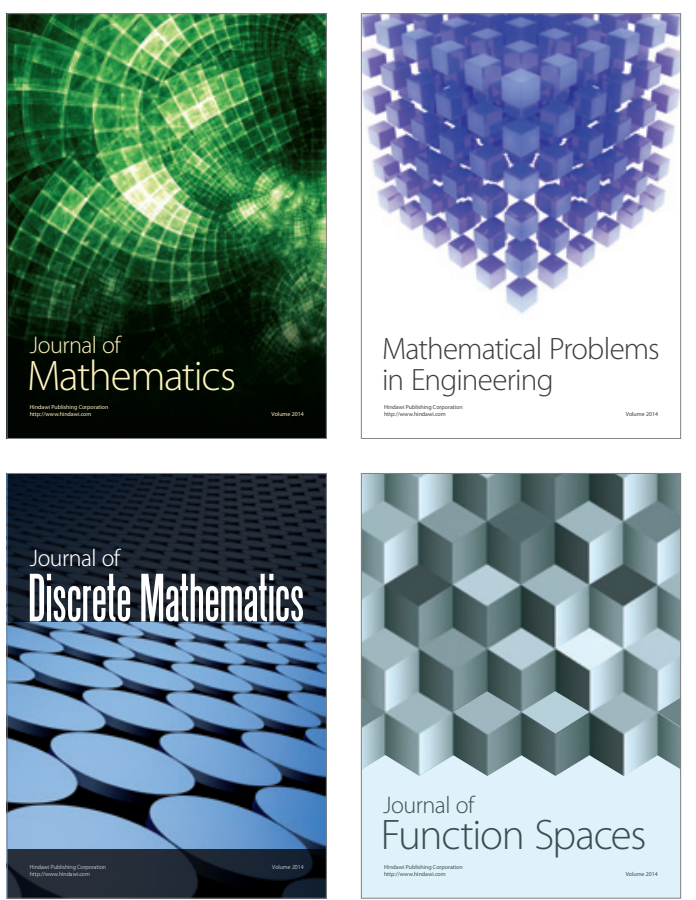

Mathematical Problems in Engineering
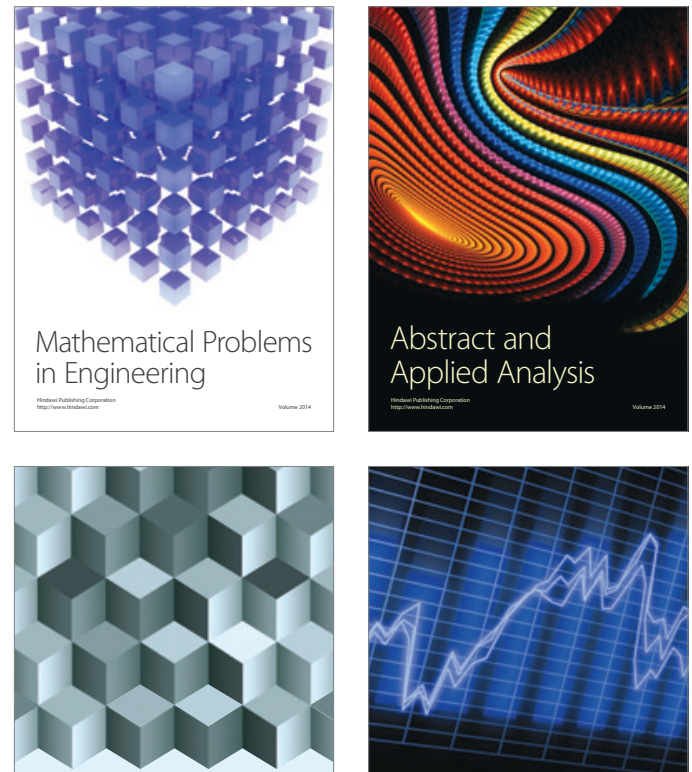

Journal of

Function Spaces

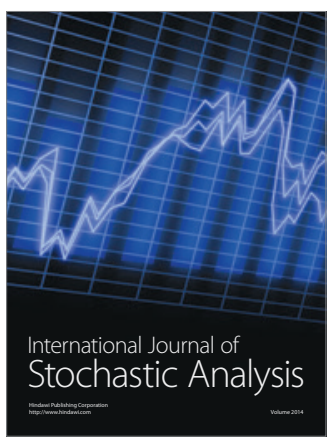

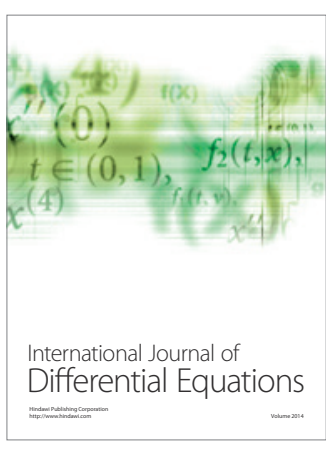
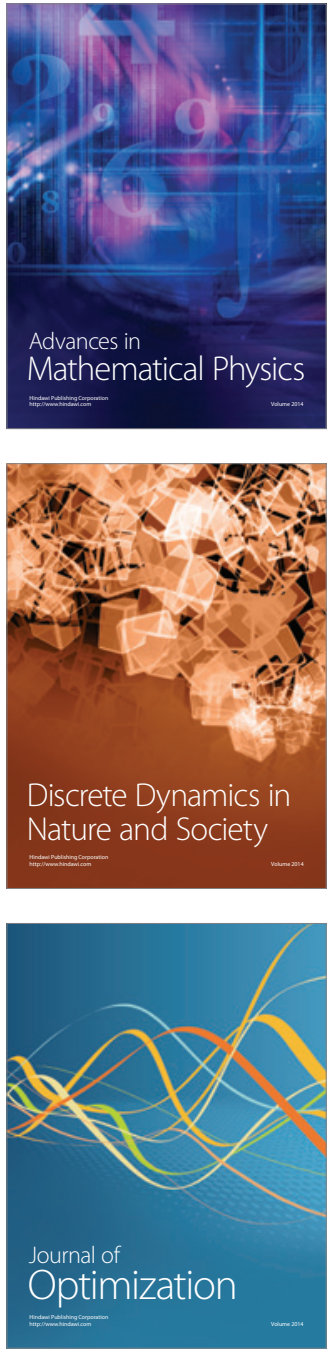